\title{
Theoretical Studies of Stereoselectivities of Intramolecular Aldol Cyclizations Catalyzed by Amino Acids
}

\author{
Fernando R. Clemente and K. N. Houk* \\ Department of Chemistry and Biochemistry, University of California, Los Angeles 90095-1569.
}

\section{SUPPORTING INFORMATION}

1) Full citation of GAUSSIAN software (Reference 26).

2) Cartesian coordinates (in $\AA$ ) of all the structures with their computed electronic energies (SCF in Hartree) and unscaled zero-point energy corrections (ZPE in Hartree).

\section{1) Full citation of GAUSSIAN software (Reference 26).}

Frisch, M. J.; Trucks, G. W.; Schlegel, H. B.; Scuseria, G. E.; Robb, M. A.; Cheeseman, J. R.; Zakrzewski, V. G.; Montgomery, J. A., Jr.; Stratmann, R. E.; Burant, J. C.; Dapprich, S.; Millam, J. M.; Daniels, A. D.; Kudin, K. N.; Strain, M. C.; Farkas, O.; Tomasi, J.; Barone, V.; Cossi, M.; Cammi, R.; Mennucci, B.; Pomelli, C.; Adamo, C.; Clifford, S.; Ochterski, J.; Petersson, G. A.; Ayala, P. Y.; Cui, Q.; Morokuma, K.; Malick, D. K.; Rabuck, A. D.; Raghavachari, K.; Foresman, J. B.; Cioslowski, J.; Ortiz, J. V.; Baboul, A. G.; Stefanov, B. B.; Liu, G.; Liashenko, A.; Piskorz, P.; Komaromi, I.; Gomperts, R.; Martin, R. L.; Fox, D. J.; Keith, T.; Al-Laham, M. A.; Peng, C. Y.; Nanayakkara, A.; Challacombe, M.; Gill, P. M. W.; Johnson, B.; Chen, W.; Wong, M. W.; Andres, J. L.; Gonzalez, C.; Head-Gordon, M.; Replogle, E. S.; Pople, J. A. Gaussian 98, Revision A.9; Gaussian, Inc.: Pittsburgh, PA, 1998. 
2) Cartesian coordinates (in $\AA$ ) of all the structures with their computed electronic energies (SCF in Hartree) and unscaled zero-point energy corrections (ZPE in Hartree).

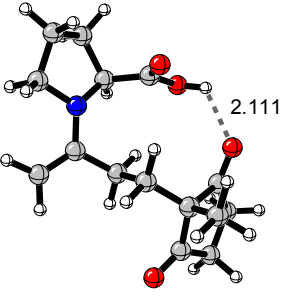

anti-a (O-H-O)

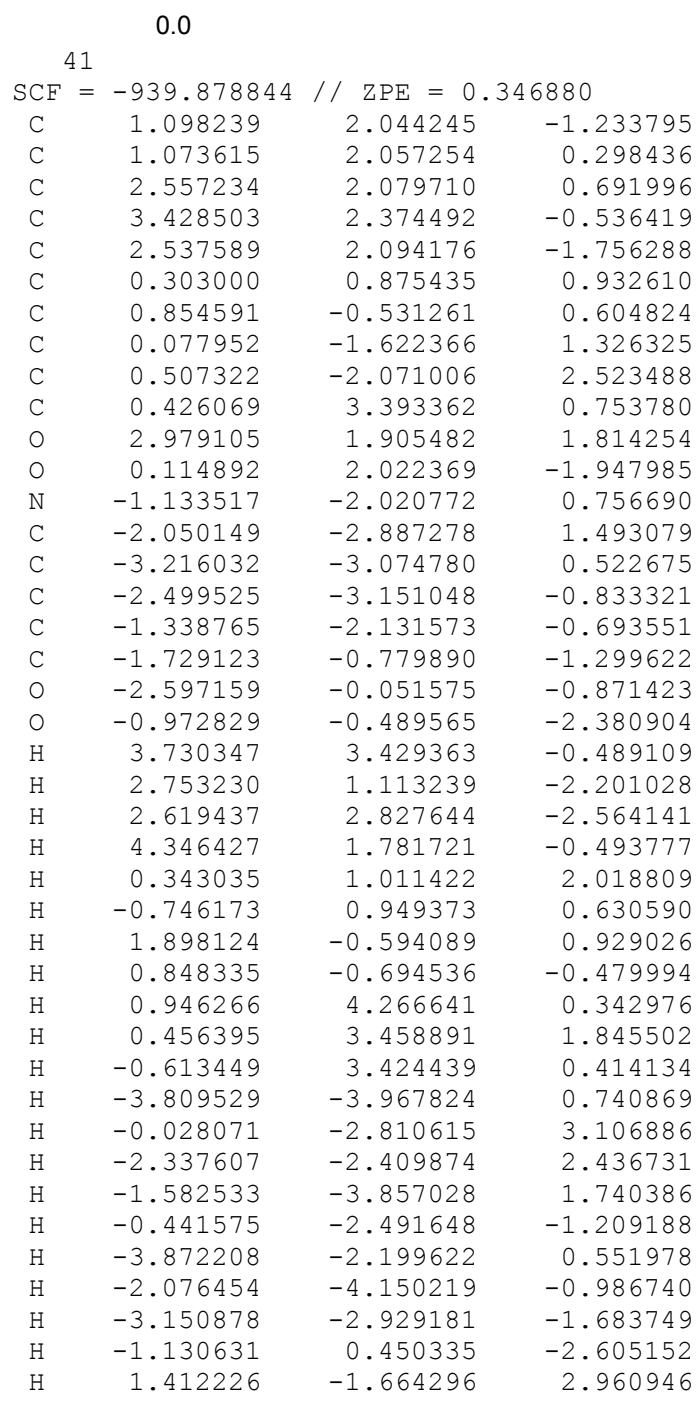

$(S, S)-4$

$E_{\text {rel }}=0.0 \mathrm{kcal} / \mathrm{mol}$

$\left(\mathrm{E}_{\mathrm{act}}=10.5 \mathrm{kcal} / \mathrm{mol}\right)$

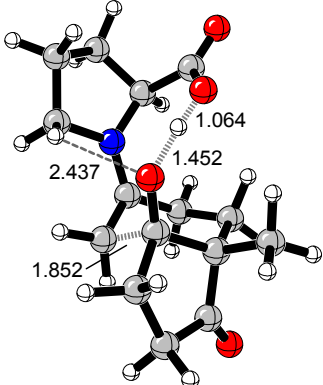

41

$\mathrm{SCF}=-939.8627052 / / \mathrm{ZPE}=0.347407$

$\begin{array}{llll}\text { C } & 3.824856 & -0.476687 & 0.576412\end{array}$

$\begin{array}{llll}\mathrm{C} & 3.461576 & -1.551431 & -0.459029\end{array}$

$\begin{array}{llll}\mathrm{C} & 2.016403 & -1.936786 & -0.105979\end{array}$

$\begin{array}{llll}\mathrm{N} & 1.486148 & -0.751738 & 0.636254\end{array}$

$\begin{array}{llll}\mathrm{C} & 2.509782 & 0.306234 & 0.717386\end{array}$

C $\quad 0.262224 \quad-0.701053 \quad 1.138829$

$\begin{array}{llll}\mathrm{C} & -0.255562 & 0.495414 & 1.887947\end{array}$

C $\quad-0.998521 \quad 1.441350 \quad 0.899140$

$\begin{array}{llll}\mathrm{C} & -1.891840 & 0.713611 & -0.114756\end{array}$

$\begin{array}{llll}\mathrm{C} & -1.278169 & -0.571616 & -0.778531\end{array}$

C $\quad-2.510409 \quad-1.381979 \quad-1.275641$

C $\quad-3.666054 \quad-1.036200 \quad-0.317818$

C $-3.191540 \quad 0.182363 \quad 0.489483$

$\begin{array}{llll}0 & -0.270940 & -0.453810 & -1.589449\end{array}$

$\begin{array}{llll}0 & -3.751453 & 0.629016 & 1.470108\end{array}$

C $\quad-2.271249 \quad 1.710854 \quad-1.242125$

C $\quad 2.410608 \quad 1.398228 \quad-0.395140$

$\begin{array}{llll}\text { O } & 1.475734 & 1.319541 & -1.314772\end{array}$

C $\quad-0.746414 \quad-1.590657 \quad 0.673995$

$\begin{array}{llll}0 & 3.240510 & 2.285734 & -0.347592\end{array}$

$\mathrm{H} \quad 0.527916 \quad 1.048167 \quad 2.411725$

$\begin{array}{llll}\mathrm{H} & -0.965878 & 0.137349 & 2.639512\end{array}$

$\mathrm{H} \quad-0.262845 \quad 2.039523 \quad 0.355788$

$\begin{array}{rrrr}\mathrm{H} & -0.262845 & 2.039523 & 0.355788 \\ \mathrm{H} & -0.439577 & -2.524294 & 0.213712\end{array}$

$\mathrm{H} \quad-1.590254 \quad-1.713271 \quad 1.349773$

$\begin{array}{rrrr}\mathrm{H} & -1.590254 & -1.713271 & 1.349773 \\ \mathrm{H} & 1.971305 & -2.803457 & 0.564389 \\ \mathrm{H} & 1.399647 & -2.121217 & -0.983721\end{array}$

$\mathrm{H}-2.296521 \quad-2.452532 \quad-1.337680$

$\mathrm{H} \quad-2.715260 \quad-1.040535 \quad-2.296220$

$\mathrm{H}-4.596758 \quad-0.783786 \quad-0.839408$

$\mathrm{H} \quad-3.008794 \quad 1.292900 \quad-1.935231$

$\begin{array}{lll}-1.618196 & 2.127623 & 1.486408\end{array}$

$-2.692979 \quad 2.625643 \quad-0.812317$

$2.447171 \quad 0.830247 \quad 1.672220$

$\begin{array}{lll}4.637326 & 0.182761 & 0.267956\end{array}$

$3.499811-1.128475-1.468328$

$4.089684-0.933872 \quad 1.537223$

$\begin{array}{lll}4.128520 & -2.417320 & -0.433488\end{array}$

$\begin{array}{lll}-3.918652 & -1.840198 & 0.382550\end{array}$

$\begin{array}{lll}-1.374741 & 1.961008 & -1.816115\end{array}$

$0.771083 \quad 0.522180 \quad-1.323921$ 


\section{(S,S)-4 - B3LYP/6-31+G(d,p) optimized}

\begin{tabular}{|c|c|c|c|}
\hline $\mathrm{SCF}$ & $=-939.9324342$ & $/ / \mathrm{ZPE}=$ & 45410 \\
\hline $\mathrm{C}$ & 3.835003 & -0.462840 & 0.577422 \\
\hline $\mathrm{C}$ & 3.498198 & -1.548373 & -0.456588 \\
\hline $\mathrm{C}$ & 2.048513 & -1.941647 & -0.130897 \\
\hline $\mathrm{N}$ & 1.498530 & -0.764082 & 0.606484 \\
\hline $\mathrm{C}$ & 2.507887 & 0.305280 & 0.709143 \\
\hline $\mathrm{C}$ & 0.270185 & -0.736123 & 1.111917 \\
\hline $\mathrm{C}$ & -0.251299 & 0.440304 & 1.891770 \\
\hline $\mathrm{C}$ & -1.001538 & 1.413324 & 0.937945 \\
\hline $\mathrm{C}$ & -1.906215 & 0.718397 & -0.089406 \\
\hline $\mathrm{C}$ & -1.293409 & -0.524369 & -0.819880 \\
\hline $\mathrm{C}$ & -2.507022 & -1.345973 & -1.322649 \\
\hline $\mathrm{C}$ & -3.655836 & -1.053387 & -0.339155 \\
\hline $\mathrm{C}$ & -3.195381 & 0.143589 & 0.501240 \\
\hline O & -0.280078 & -0.381281 & -1.609934 \\
\hline O & -3.767844 & 0.559155 & 1.490691 \\
\hline $\mathrm{C}$ & -2.325943 & 1.761892 & -1.163867 \\
\hline $\mathrm{C}$ & 2.402764 & 1.413454 & -0.383496 \\
\hline $\mathrm{O}$ & 1.503678 & 1.319193 & -1.335073 \\
\hline C & -0.722894 & -1.634238 & 0.653231 \\
\hline O & 3.197991 & 2.333995 & -0.297044 \\
\hline $\mathrm{H}$ & 0.532757 & 0.981490 & 2.425115 \\
\hline $\mathrm{H}$ & -0.953383 & 0.059077 & 2.638883 \\
\hline $\mathrm{H}$ & -0.273608 & 2.028142 & 0.402195 \\
\hline $\mathrm{H}$ & -0.421444 & -2.539626 & 0.139235 \\
\hline $\mathrm{H}$ & -1.571269 & -1.771910 & 1.317696 \\
\hline $\mathrm{H}$ & 1.993972 & -2.812032 & 0.533665 \\
\hline $\mathrm{H}$ & 1.454422 & -2.131261 & -1.024156 \\
\hline $\mathrm{H}$ & -2.268328 & -2.407609 & -1.419868 \\
\hline $\mathrm{H}$ & -2.733222 & -0.979395 & -2.330056 \\
\hline $\mathrm{H}$ & -4.596584 & -0.796118 & -0.838660 \\
\hline $\mathrm{H}$ & -3.050879 & 1.355975 & -1.875691 \\
\hline $\mathrm{H}$ & -1.613068 & 2.086446 & 1.547718 \\
\hline $\mathrm{H}$ & -2.777471 & 2.633320 & -0.679730 \\
\hline $\mathrm{H}$ & 2.432495 & 0.816024 & 1.669821 \\
\hline $\mathrm{H}$ & 4.646811 & 0.200463 & 0.276597 \\
\hline $\mathrm{H}$ & 3.557147 & -1.136785 & -1.468969 \\
\hline $\mathrm{H}$ & 4.092640 & -0.912058 & 1.543458 \\
\hline $\mathrm{H}$ & 4.170477 & -2.408577 & -0.406311 \\
\hline $\mathrm{H}$ & -3.885856 & -1.884233 & 0.336228 \\
\hline $\mathrm{H}$ & -1.443980 & 2.079039 & -1.726106 \\
\hline $\mathrm{H}$ & 0.770608 & 0.543979 & -1.355951 \\
\hline
\end{tabular}

$(R, R)-4$

$\mathrm{E}_{\mathrm{rel}}=3.3 \mathrm{kcal} / \mathrm{mol}$

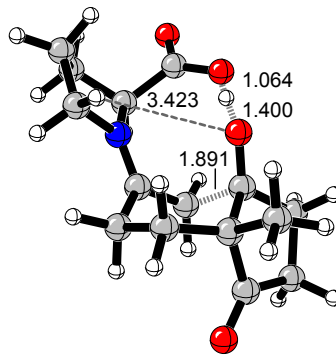

41

\begin{tabular}{|c|c|c|c|}
\hline C & -3.515674 & -1.544723 & 0.701456 \\
\hline $\mathrm{C}$ & -2.037508 & -1.919096 & 0.537949 \\
\hline $\mathrm{N}$ & -1.474929 & -0.829635 & -0.301339 \\
\hline $\mathrm{C}$ & -2.547451 & 0.116029 & -0.729495 \\
\hline C & -3.810490 & -0.747575 & -0.575217 \\
\hline $\mathrm{C}$ & -2.742168 & 1.495001 & -0.003656 \\
\hline O & -1.775189 & 2.060179 & 0.666173 \\
\hline $\mathrm{C}$ & -0.246048 & -0.846883 & -0.802171 \\
\hline $\mathrm{C}$ & 0.379641 & 0.336994 & -1.267637 \\
\hline O & -3.821470 & 2.028321 & -0.195429 \\
\hline $\mathrm{C}$ & 0.679164 & -2.016841 & -0.601224 \\
\hline $\mathrm{C}$ & 1.640817 & -1.750044 & 0.586452 \\
\hline $\mathrm{C}$ & 2.262924 & -0.352473 & 0.569558 \\
\hline $\mathrm{C}$ & 1.229244 & 0.796040 & 0.358404 \\
\hline $\mathrm{C}$ & 2.073978 & 2.012317 & -0.119599 \\
\hline $\mathrm{C}$ & 3.283266 & 1.408717 & -0.865368 \\
\hline $\mathrm{C}$ & 3.271496 & -0.097300 & -0.544944 \\
\hline O & 0.322564 & 0.923184 & 1.269631 \\
\hline $\mathrm{C}$ & 2.982343 & -0.115466 & 1.924605 \\
\hline O & 3.936306 & -0.939336 & -1.114523 \\
\hline $\mathrm{H}$ & 2.384569 & 2.551111 & 0.782934 \\
\hline $\mathrm{H}$ & 1.491417 & 2.715260 & -0.721030 \\
\hline $\mathrm{H}$ & 1.154764 & 0.191802 & -2.016301 \\
\hline $\mathrm{H}$ & 0.144880 & -2.957030 & -0.443912 \\
\hline $\mathrm{H}$ & 1.275263 & -2.125770 & -1.513184 \\
\hline $\mathrm{H}$ & -0.241045 & 1.209021 & -1.453417 \\
\hline $\mathrm{H}$ & -2.357516 & 0.375399 & -1.775517 \\
\hline $\mathrm{H}$ & 1.091571 & -1.865442 & 1.527650 \\
\hline $\mathrm{H}$ & 3.682141 & -0.932594 & 2.130576 \\
\hline $\mathrm{H}$ & 2.231216 & -0.066766 & 2.718168 \\
\hline $\mathrm{H}$ & 3.547478 & 0.822142 & 1.938683 \\
\hline $\mathrm{H}$ & 2.432682 & -2.506579 & 0.565962 \\
\hline $\mathrm{H}$ & -1.488861 & -1.973019 & 1.481813 \\
\hline $\mathrm{H}$ & -1.932681 & -2.875790 & 0.014375 \\
\hline $\mathrm{H}$ & -4.147284 & -2.429273 & 0.819887 \\
\hline $\mathrm{H}$ & -4.695445 & -0.115630 & -0.513209 \\
\hline $\mathrm{H}$ & -3.912089 & -1.422773 & -1.433556 \\
\hline $\mathrm{H}$ & -3.655308 & -0.909002 & 1.582767 \\
\hline $\mathrm{H}$ & 3.244239 & 1.534876 & -1.952896 \\
\hline $\mathrm{H}$ & 4.240181 & 1.836911 & -0.544076 \\
\hline $\mathrm{H}$ & -0.884567 & 1.523624 & 0.89217 \\
\hline
\end{tabular}

B3LYP/ 6-31+G $(d, p) / / B 3 L Y P / 6-31 G(d)=-939.9273696$ 


\section{$(R, R)-4$ - B3LYP/6-31+G(d,p) optimized}

\begin{tabular}{crrr} 
& 41 & & \\
$\mathrm{SCF}$ & - & -939.9278762 & \multicolumn{1}{l}{$/$ / ZPE $=0.344866$} \\
$\mathrm{C}$ & -3.551228 & -1.539237 & 0.670494 \\
$\mathrm{C}$ & -2.066170 & -1.903762 & 0.550149 \\
$\mathrm{~N}$ & -1.489445 & -0.820809 & -0.291191 \\
$\mathrm{C}$ & -2.551690 & 0.121203 & -0.741177 \\
$\mathrm{C}$ & -3.817279 & -0.748106 & -0.617096 \\
$\mathrm{C}$ & -2.763038 & 1.479239 & 0.007779 \\
$\mathrm{O}$ & -1.865974 & 1.962998 & 0.825890 \\
$\mathrm{C}$ & -0.253479 & -0.848347 & -0.785056 \\
$\mathrm{C}$ & 0.371034 & 0.306068 & -1.306739 \\
$\mathrm{O}$ & -3.803560 & 2.062561 & -0.253686 \\
$\mathrm{C}$ & 0.666539 & -2.015852 & -0.543377 \\
$\mathrm{C}$ & 1.625674 & -1.719389 & 0.638808 \\
$\mathrm{C}$ & 2.274915 & -0.334435 & 0.577893 \\
$\mathrm{C}$ & 1.268800 & 0.832409 & 0.349328 \\
$\mathrm{C}$ & 2.115681 & 2.011319 & -0.197210 \\
$\mathrm{C}$ & 3.305394 & 1.360891 & -0.933347 \\
$\mathrm{C}$ & 3.284569 & -0.125409 & -0.547185 \\
$\mathrm{O}$ & 0.363136 & 1.013664 & 1.244486 \\
$\mathrm{C}$ & 3.020166 & -0.077961 & 1.918584 \\
$\mathrm{O}$ & 3.959934 & -0.991542 & -1.070913 \\
$\mathrm{H}$ & 2.446310 & 2.585201 & 0.676101 \\
$\mathrm{H}$ & 1.529509 & 2.692898 & -0.817894 \\
$\mathrm{H}$ & 1.157740 & 0.126665 & -2.033652 \\
$\mathrm{H}$ & 0.126794 & -2.945917 & -0.355010 \\
$\mathrm{H}$ & 1.261380 & -2.160177 & -1.450654 \\
$\mathrm{H}$ & -0.230114 & 1.184695 & -1.518355 \\
$\mathrm{H}$ & -2.346062 & 0.392140 & -1.780483 \\
$\mathrm{H}$ & 1.070553 & -1.787889 & 1.580860 \\
$\mathrm{H}$ & 3.725486 & -0.891401 & 2.115556 \\
$\mathrm{H}$ & 2.286497 & -0.024533 & 2.727329 \\
$\mathrm{H}$ & 3.580684 & 0.861460 & 1.910710 \\
$\mathrm{H}$ & 2.404664 & -2.488614 & 0.654405 \\
$\mathrm{H}$ & -1.545196 & -1.943696 & 1.510176 \\
$\mathrm{H}$ & -1.942431 & -2.865740 & 0.041617 \\
$\mathrm{H}$ & -4.178933 & -2.428212 & 0.771252 \\
$\mathrm{H}$ & -4.712313 & -0.128244 & -0.583936 \\
$\mathrm{H}$ & -3.885918 & -1.425486 & -1.476472 \\
$\mathrm{H}$ & -3.722405 & -0.904297 & 1.546210 \\
$\mathrm{H}$ & 3.251273 & 1.439261 & -2.024216 \\
$\mathrm{H}$ & 4.272797 & 1.787472 & -0.644923 \\
$\mathrm{H}$ & -0.929612 & 1.497529 & 0.958051
\end{tabular}

$(R, S)-7$

$E_{\text {rel }}=0.0 \mathrm{kcal} / \mathrm{mol}$

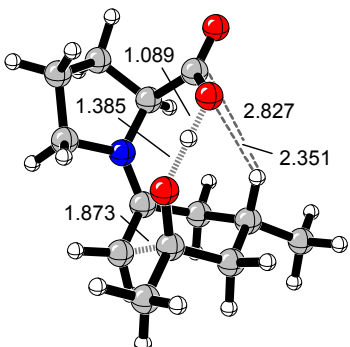

38

\begin{tabular}{crrr}
$\mathrm{SCF}$ & -188.431655 & \multicolumn{1}{c}{$/ / \mathrm{ZPE}=0.329537$} \\
$\mathrm{C}$ & -3.211677 & -0.173497 & -0.815048 \\
$\mathrm{C}$ & -3.221636 & -0.190051 & 0.720518 \\
$\mathrm{C}$ & -1.723158 & -0.219864 & 1.062147 \\
$\mathrm{~N}$ & -1.135985 & 0.663177 & 0.036415 \\
$\mathrm{C}$ & -2.015389 & 0.723650 & -1.167863 \\
$\mathrm{C}$ & 0.005267 & 1.330987 & 0.126312 \\
$\mathrm{C}$ & 0.905360 & 1.206579 & 1.324875 \\
$\mathrm{C}$ & 1.995836 & 0.113402 & 1.064978 \\
$\mathrm{C}$ & 2.602172 & 0.260359 & -0.339276 \\
$\mathrm{C}$ & 1.566292 & 0.316508 & -1.488578 \\
$\mathrm{O}$ & 0.728567 & -0.681488 & -1.592430 \\
$\mathrm{C}$ & -1.196456 & -1.692307 & 1.050285 \\
$\mathrm{O}$ & -0.290634 & -2.056660 & 0.179696 \\
$\mathrm{C}$ & 2.194951 & 0.719526 & -2.829275 \\
$\mathrm{C}$ & 3.064251 & 0.170918 & 2.162750 \\
$\mathrm{C}$ & 0.619779 & 1.867874 & -1.036639 \\
$\mathrm{O}$ & -1.688641 & -2.434926 & 1.881193 \\
$\mathrm{H}$ & 0.365517 & 0.974743 & 2.246543 \\
$\mathrm{H}$ & 1.404673 & 2.171628 & 1.472427 \\
$\mathrm{H}$ & 3.247216 & -0.607172 & -0.534375 \\
$\mathrm{H}$ & 3.246692 & 1.150267 & -0.376793 \\
$\mathrm{H}$ & 1.504553 & -0.861829 & 1.115959 \\
$\mathrm{H}$ & 0.000065 & 2.069223 & -1.904569 \\
$\mathrm{H}$ & 1.362711 & 2.640481 & -0.855187 \\
$\mathrm{H}$ & -1.528096 & 0.172595 & 2.061512 \\
$\mathrm{H}$ & 1.415991 & 0.806182 & -3.592160 \\
$\mathrm{H}$ & 2.878909 & -0.079009 & -3.143142 \\
$\mathrm{H}$ & 2.758873 & 1.657236 & -2.775724 \\
$\mathrm{H}$ & -2.313136 & 1.768035 & -1.322615 \\
$\mathrm{H}$ & -1.461595 & 0.369942 & -2.036305 \\
$\mathrm{H}$ & -4.142141 & 0.202520 & -1.248967 \\
$\mathrm{H}$ & -3.676247 & 0.725992 & 1.116651 \\
$\mathrm{H}$ & -3.046282 & -1.185363 & -1.199449 \\
$\mathrm{H}$ & -3.733120 & -1.050395 & 1.154095 \\
$\mathrm{H}$ & 0.133332 & -1.357418 & -0.540016 \\
$\mathrm{H}$ & 3.805805 & -0.623177 & 2.022693 \\
$\mathrm{H}$ & 2.622267 & 0.040568 & 3.157696 \\
$\mathrm{H}$ & 3.595340 & 1.131595 & 2.151758 \\
& & &
\end{tabular}


$(S, R)-7$

$\mathrm{E}_{\text {rel }}=1.1 \mathrm{kcal} / \mathrm{mol}$

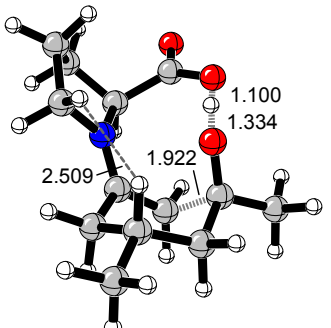

38

\begin{tabular}{crrr}
$\mathrm{SCF}=$ & -788.429100 & \multicolumn{1}{c}{$/ \mathrm{ZPE}=0.328789$} \\
$\mathrm{C}$ & -1.580460 & 0.630252 & -2.859392 \\
$\mathrm{C}$ & -1.568929 & 0.599696 & -1.320969 \\
$\mathrm{~N}$ & -0.110223 & 0.589316 & -1.022204 \\
$\mathrm{C}$ & 0.669737 & 0.121995 & -2.195256 \\
$\mathrm{C}$ & -0.399073 & -0.279627 & -3.219819 \\
$\mathrm{C}$ & -2.496461 & -0.556834 & -0.801888 \\
$\mathrm{O}$ & -2.080313 & -1.432565 & 0.065153 \\
$\mathrm{C}$ & 0.436372 & 1.096250 & 0.076634 \\
$\mathrm{C}$ & -0.284571 & 1.164013 & 1.292140 \\
$\mathrm{C}$ & 1.928235 & 1.271568 & 0.190162 \\
$\mathrm{C}$ & 2.581413 & 0.077219 & 0.952588 \\
$\mathrm{C}$ & 4.061928 & 0.366284 & 1.220207 \\
$\mathrm{C}$ & 1.795972 & -0.228245 & 2.233908 \\
$\mathrm{C}$ & 0.320854 & -0.544477 & 1.931624 \\
$\mathrm{O}$ & 0.173561 & -1.426373 & 0.980912 \\
$\mathrm{C}$ & -0.570400 & -0.717445 & 3.164098 \\
$\mathrm{O}$ & -3.631472 & -0.525139 & -1.251126 \\
$\mathrm{H}$ & -0.512165 & 0.129242 & 3.856633 \\
$\mathrm{H}$ & -0.247241 & -1.622618 & 3.694528 \\
$\mathrm{H}$ & -1.609417 & -0.867053 & 2.857031 \\
$\mathrm{H}$ & 0.069062 & 1.884958 & 2.024103 \\
$\mathrm{H}$ & 2.226815 & -1.119849 & 2.710238 \\
$\mathrm{H}$ & 1.884115 & 0.595766 & 2.955365 \\
$\mathrm{H}$ & 2.409135 & 1.399774 & -0.783400 \\
$\mathrm{H}$ & 1.288676 & 0.942986 & -2.576173 \\
$\mathrm{H}$ & 2.110569 & 2.188192 & 0.765091 \\
$\mathrm{H}$ & -1.365496 & 1.064132 & 1.263257 \\
$\mathrm{H}$ & 1.322210 & -0.702503 & -1.897300 \\
$\mathrm{H}$ & 2.497606 & -0.810537 & 0.316300 \\
$\mathrm{H}$ & -1.991137 & 1.513787 & -0.893920 \\
$\mathrm{H}$ & -2.542944 & 0.289319 & -3.239601 \\
$\mathrm{H}$ & -0.045631 & -0.151777 & -4.246691 \\
$\mathrm{H}$ & -0.677853 & -1.330579 & -3.085580 \\
$\mathrm{H}$ & -1.399266 & 1.651012 & -3.217879 \\
$\mathrm{H}$ & -1.045527 & -1.416785 & 0.438856 \\
$\mathrm{H}$ & 4.608694 & 0.556591 & 0.288563 \\
$\mathrm{H}$ & 4.186003 & 1.244959 & 1.866255 \\
$\mathrm{H}$ & 4.540489 & -0.483989 & 1.718885 \\
& & & \\
& & & \\
& & &
\end{tabular}

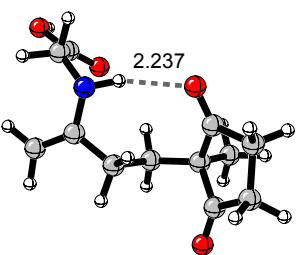

syn-b

0.0
34

$\mathrm{SCF}=-823.149420 / / \mathrm{ZPE}=0.282340$

1. 785991

1. 818502

0.522530

0.090164

$-1.404096$

$-2.217680$

$-1.811220$

$-3.176876$

$-3.592712$

$-2.842387$

$-4.941384$

$-5.124776$

$-3.295886$

$-1.800428$

0.588211

0.400261

$-0.272267$

0.652695

3. 061972

3. 316846

3. 943858

3. 188977

2. 120273

4. 936904

4. 088634

3. 653325

3. 113019

3. 037579

1. 287283

2. 238713

$-1.135962$

$-3.332573$

$-3.861684$
$-1.709294$

$-1.151771$

$-0.213551$

0.582272

1. 449158

1. 385296

2. 411040

0.165769

$-0.265750$

$-0.657518$

$-0.916857$

$-0.737679$

$-1.033722$

2. 375254

3. 338824

1.099742

2. 486534

$-0.129327$

1. 225367

0.646460

1. 688168

$-0.007461$

$-1.264505$

$-1.076533$

$-0.215188$

0.722682

$-2.186335$

$-1.366457$

$-1.667382$

$-1.762403$

$-0.416944$

$-0.584201$

$-1.147711$

0.500804
$-1.020024$

$-0.584544$

0.622365

0.826037

$-0.390224$

$-0.615738$

$-0.308602$

$-1.170395$

$-1.055358$

0.364453

1. 277472

0.473978

1. 385797

$-0.423417$

0.064887

$-1.305666$

$-0.242753$

1.072760

1. 702518

0.360992

0.923196

$-0.715562$

$-1.190086$

1.882673

$-0.303155$

$-1.520359$

$-0.816224$

$-2.276126$

1.777497

2.066555

2. 747967

$-1.051732$

$-1.689612$

$-1.430469$ 


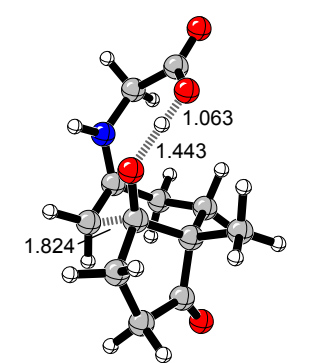

anti-8

$\mathrm{E}_{\mathrm{rel}}=0.0 \mathrm{kcal} / \mathrm{mo}$

$\left(E_{\text {act }}=12.8 \mathrm{kcal} / \mathrm{mol}\right)$

34

$\mathrm{SCF}=-823.129213 / / \mathrm{ZPE}=0.282546$

\begin{tabular}{|c|c|c|c|}
\hline $\mathrm{C}$ & -1.239553 & -0.346086 & -0.329967 \\
\hline $\mathrm{C}$ & -1.247532 & -0.334215 & 1.493798 \\
\hline $\mathrm{C}$ & 0.159284 & -0.264659 & 1.679427 \\
\hline $\mathrm{C}$ & 0.783372 & 1.080939 & 1.486716 \\
\hline $\mathrm{C}$ & 0.736612 & 1.393206 & -0.042855 \\
\hline $\mathrm{C}$ & -0.598680 & 1.040675 & -0.716713 \\
\hline 0 & -0.672362 & -1.454105 & -0.707148 \\
\hline $\mathrm{H}$ & 0.761821 & -1.540945 & -0.844349 \\
\hline 0 & 1.786284 & -1.654148 & -1.104607 \\
\hline $\mathrm{C}$ & 2.695492 & -1.746754 & -0.162319 \\
\hline 0 & 3.876094 & -1.945553 & -0.360330 \\
\hline $\mathrm{C}$ & 2.275525 & -1.566871 & 1.327880 \\
\hline $\mathrm{N}$ & 0.854652 & -1.392880 & 1.601934 \\
\hline $\mathrm{H}$ & -1.730952 & -1.284676 & 1.714637 \\
\hline $\mathrm{H}$ & -1.806760 & 0.521782 & 1.866522 \\
\hline $\mathrm{H}$ & 1.812606 & 1.141676 & 1.850088 \\
\hline $\mathrm{H}$ & 0.192624 & 1.825028 & 2.027855 \\
\hline $\mathrm{H}$ & 1.541699 & 0.849350 & -0.544089 \\
\hline $\mathrm{H}$ & 0.923714 & 2.464365 & -0.173547 \\
\hline $\mathrm{C}$ & -1.716466 & 2.025228 & -0.367403 \\
\hline 0 & -1.557917 & 3.181755 & -0.033140 \\
\hline $\mathrm{C}$ & -3.074139 & 1.314466 & -0.466040 \\
\hline $\mathrm{C}$ & -2.753813 & -0.179831 & -0.653455 \\
\hline $\mathrm{C}$ & -0.400976 & 1.077533 & -2.255385 \\
\hline $\mathrm{H}$ & -3.644985 & 1.740356 & -1.299469 \\
\hline $\mathrm{H}$ & -3.648786 & 1.536421 & 0.440328 \\
\hline $\mathrm{H}$ & -2.884144 & -0.493679 & -1.694620 \\
\hline $\mathrm{H}$ & -3.374552 & -0.842300 & -0.043821 \\
\hline $\mathrm{H}$ & -1.344244 & 0.948099 & -2.795855 \\
\hline $\mathrm{H}$ & 0.033610 & 2.035727 & -2.559389 \\
\hline $\mathrm{H}$ & 0.270579 & 0.267490 & -2.553474 \\
\hline $\mathrm{H}$ & 0.287749 & -2.228474 & 1.488101 \\
\hline $\mathrm{H}$ & 2.832101 & -0.713355 & 1.717904 \\
\hline 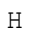 & 2.626005 & -2.449522 & 1.868840 \\
\hline
\end{tabular}

B $3 L Y P / 6-31+G(d, p) / / B 3 L Y P / 6-31 G(d)=-823.1950163$ anti-8 - B3LYP/6-31+G(d,p) optimized

\begin{tabular}{lrrr} 
& 34 & & \\
$\mathrm{SCF}$ & - & -823.1954275 & \multicolumn{1}{c}{$/ \mathrm{ZPE}=0.281052$} \\
$\mathrm{C}$ & -0.875174 & 0.280470 & -0.970014 \\
$\mathrm{C}$ & -0.630015 & -1.615224 & -0.987943 \\
$\mathrm{C}$ & 0.498408 & -1.622915 & -0.141031 \\
$\mathrm{C}$ & 0.233253 & -1.452637 & 1.323082 \\
$\mathrm{C}$ & -0.184032 & 0.030210 & 1.567231 \\
$\mathrm{C}$ & -1.183691 & 0.581350 & 0.539447 \\
$\mathrm{O}$ & 0.164531 & 0.789029 & -1.545484 \\
$\mathrm{H}$ & 1.405771 & 1.039818 & -0.883540 \\
$\mathrm{O}$ & 2.307860 & 1.389551 & -0.447060 \\
$\mathrm{C}$ & 3.182754 & 0.556190 & 0.063231 \\
$\mathrm{O}$ & 4.216289 & 0.898578 & 0.607710 \\
$\mathrm{C}$ & 2.922126 & -0.975078 & -0.009417 \\
$\mathrm{~N}$ & 1.701846 & -1.414113 & -0.673256 \\
$\mathrm{H}$ & -0.471563 & -1.776558 & -2.051809 \\
$\mathrm{H}$ & -1.523904 & -2.092350 & -0.596030 \\
$\mathrm{H}$ & 1.091691 & -1.704470 & 1.950036 \\
$\mathrm{H}$ & -0.590440 & -2.112798 & 1.606743 \\
$\mathrm{H}$ & 0.707379 & 0.663888 & 1.566009 \\
$\mathrm{H}$ & -0.632038 & 0.096435 & 2.563909 \\
$\mathrm{C}$ & -2.596981 & 0.014238 & 0.698534 \\
$\mathrm{O}$ & -3.079780 & -0.394212 & 1.737393 \\
$\mathrm{C}$ & -3.311405 & 0.019860 & -0.657378 \\
$\mathrm{C}$ & -2.239012 & 0.391864 & -1.698076 \\
$\mathrm{C}$ & -1.283807 & 2.122196 & 0.721630 \\
$\mathrm{H}$ & -4.141503 & 0.734062 & -0.618982 \\
$\mathrm{H}$ & -3.763484 & -0.965027 & -0.815786 \\
$\mathrm{H}$ & -2.332260 & 1.434501 & -2.020706 \\
$\mathrm{H}$ & -2.268114 & -0.221036 & -2.602074 \\
$\mathrm{H}$ & -2.051379 & 2.564496 & 0.079869 \\
$\mathrm{H}$ & -1.531312 & 2.360354 & 1.760652 \\
$\mathrm{H}$ & -0.325908 & 2.581852 & 0.465000 \\
$\mathrm{H}$ & 1.723182 & -1.383112 & -1.686973 \\
$\mathrm{H}$ & 2.949520 & -1.354675 & 1.012565 \\
$\mathrm{H}$ & 3.775708 & -1.413305 & -0.532328 \\
& & &
\end{tabular}




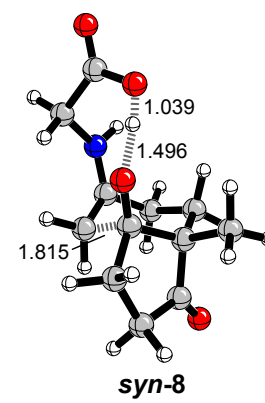

$\mathrm{E}_{\mathrm{rel}}=1.7 \mathrm{kcal} / \mathrm{mol}$

34

\begin{tabular}{crrr}
$\mathrm{SCF}$ & -123.126983 & \multicolumn{1}{c}{$/ \mathrm{ZPE}=0.283071$} \\
$\mathrm{C}$ & -0.099277 & 0.367734 & 2.653881 \\
$\mathrm{C}$ & -0.106012 & 0.359045 & 1.091919 \\
$\mathrm{C}$ & 1.425638 & 0.369794 & 0.697778 \\
$\mathrm{C}$ & 2.075360 & -0.416977 & 1.836820 \\
$\mathrm{C}$ & 1.207437 & -0.311479 & 3.097690 \\
$\mathrm{C}$ & 1.780364 & -0.201851 & -0.679937 \\
$\mathrm{C}$ & 1.160713 & -1.606294 & -0.950050 \\
$\mathrm{C}$ & -0.298405 & -1.469736 & -0.642783 \\
$\mathrm{C}$ & -0.642040 & -1.337104 & 0.729728 \\
$\mathrm{O}$ & -0.928567 & 1.178736 & 0.521707 \\
$\mathrm{~N}$ & -1.096661 & -1.140563 & -1.650098 \\
$\mathrm{C}$ & -2.433239 & -0.539246 & -1.617265 \\
$\mathrm{C}$ & -2.389757 & 0.822392 & -2.364122 \\
$\mathrm{O}$ & -3.053496 & 0.982732 & -3.366588 \\
$\mathrm{C}$ & 1.922842 & 1.835070 & 0.778931 \\
$\mathrm{O}$ & 3.101375 & -1.062272 & 1.750282 \\
$\mathrm{O}$ & -1.558106 & 1.715775 & -1.865274 \\
$\mathrm{H}$ & -1.208855 & 1.464437 & -0.919452 \\
$\mathrm{H}$ & -1.696647 & -1.316982 & 0.991497 \\
$\mathrm{H}$ & -0.070742 & -2.007476 & 1.370352 \\
$\mathrm{H}$ & 1.340727 & -1.904829 & -1.989380 \\
$\mathrm{H}$ & 1.627415 & -2.346389 & -0.295132 \\
$\mathrm{H}$ & 2.868941 & -0.289899 & -0.761694 \\
$\mathrm{H}$ & 1.437547 & 0.493086 & -1.455944 \\
$\mathrm{H}$ & 1.749956 & 0.256619 & 3.862729 \\
$\mathrm{H}$ & 1.071769 & -1.318410 & 3.509182 \\
$\mathrm{H}$ & -0.999158 & -0.100798 & 3.062123 \\
$\mathrm{H}$ & -0.129566 & 1.421226 & 2.950558 \\
$\mathrm{H}$ & 1.805046 & 2.253782 & 1.783571 \\
$\mathrm{H}$ & 1.332111 & 2.450060 & 0.093704 \\
$\mathrm{H}$ & 2.982137 & 1.898000 & 0.508093 \\
$\mathrm{H}$ & -0.678205 & -1.164208 & -2.575298 \\
$\mathrm{H}$ & -2.730645 & -0.386366 & -0.579911 \\
$\mathrm{H}$ & -3.147355 & -1.185058 & -2.132753 \\
& & &
\end{tabular}

B3LYP / 6-31+G (d,p) / / B $L Y P / 6-31 G(d)=-823.1931994$ syn-8 - B3LYP/6-31+G(d,p) optimized

\begin{tabular}{crrr} 
& 34 & & \\
$\mathrm{SCF}$ & - & -823.1937073 & \multicolumn{1}{c}{$/ \mathrm{ZPE}=0.281517$} \\
$\mathrm{C}$ & -1.773502 & -1.591205 & -1.184347 \\
$\mathrm{C}$ & -0.691528 & -0.856453 & -0.342038 \\
$\mathrm{C}$ & -1.494357 & -0.094616 & 0.779399 \\
$\mathrm{C}$ & -2.820091 & 0.225238 & 0.085375 \\
$\mathrm{C}$ & -3.068616 & -0.772448 & -1.049150 \\
$\mathrm{C}$ & -0.820200 & 1.142422 & 1.383559 \\
$\mathrm{C}$ & -0.304672 & 2.160475 & 0.324594 \\
$\mathrm{C}$ & 0.511396 & 1.381205 & -0.662621 \\
$\mathrm{C}$ & -0.200878 & 0.533554 & -1.538248 \\
$\mathrm{O}$ & 0.375269 & -1.513463 & -0.051296 \\
$\mathrm{~N}$ & 1.819918 & 1.301994 & -0.429399 \\
$\mathrm{C}$ & 2.810898 & 0.397942 & -1.014743 \\
$\mathrm{C}$ & 3.495562 & -0.442814 & 0.093325 \\
$\mathrm{O}$ & 4.699726 & -0.366258 & 0.246714 \\
$\mathrm{C}$ & -1.795167 & -1.108734 & 1.916371 \\
$\mathrm{O}$ & -3.560567 & 1.147080 & 0.373505 \\
$\mathrm{O}$ & 2.702509 & -1.182419 & 0.841988 \\
$\mathrm{H}$ & 1.726029 & -1.241218 & 0.510467 \\
$\mathrm{H}$ & 0.342079 & -0.018517 & -2.298578 \\
$\mathrm{H}$ & -1.131825 & 0.962049 & -1.900774 \\
$\mathrm{H}$ & 0.282349 & 2.944565 & 0.815279 \\
$\mathrm{H}$ & -1.152546 & 2.635596 & -0.174997 \\
$\mathrm{H}$ & -1.535912 & 1.657243 & 2.032627 \\
$\mathrm{H}$ & 0.020028 & 0.816204 & 2.007391 \\
$\mathrm{H}$ & -3.942440 & -1.384515 & -0.798579 \\
$\mathrm{H}$ & -3.339803 & -0.211048 & -1.950015 \\
$\mathrm{H}$ & -1.447886 & -1.737920 & -2.216959 \\
$\mathrm{H}$ & -1.874754 & -2.587672 & -0.741508 \\
$\mathrm{H}$ & -2.358394 & -1.975056 & 1.557467 \\
$\mathrm{H}$ & -0.850840 & -1.474846 & 2.328171 \\
$\mathrm{H}$ & -2.378093 & -0.627572 & 2.707814 \\
$\mathrm{H}$ & 2.176141 & 1.886005 & 0.319545 \\
$\mathrm{H}$ & 2.319741 & -0.262301 & -1.729630 \\
$\mathrm{H}$ & 3.589337 & 0.975823 & -1.517526
\end{tabular}




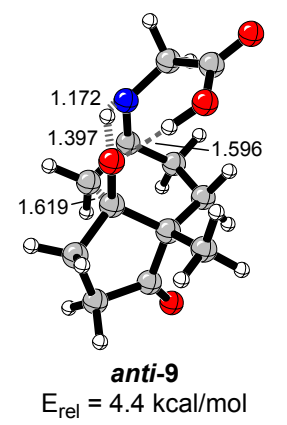

34

\begin{tabular}{crrr}
$\mathrm{SCF}$ & -823.1213006 & \multicolumn{1}{l}{$/ \mathrm{ZPE}=0.281680$} \\
$\mathrm{C}$ & 0.000000 & 0.000000 & 0.000000 \\
$\mathrm{C}$ & 0.000000 & 0.000000 & 1.618958 \\
$\mathrm{C}$ & 1.526778 & 0.000000 & 2.040392 \\
$\mathrm{C}$ & 2.454823 & 1.003548 & 1.310579 \\
$\mathrm{C}$ & 2.125703 & 1.291211 & -0.202345 \\
$\mathrm{C}$ & 0.632205 & 1.305697 & -0.243221 \\
$\mathrm{C}$ & 1.912822 & -1.460977 & 1.765327 \\
$\mathrm{C}$ & 0.672977 & -2.352813 & 1.922584 \\
$\mathrm{C}$ & -0.516030 & -1.387408 & 2.087757 \\
$\mathrm{O}$ & -0.780167 & 1.051301 & 2.040341 \\
$\mathrm{C}$ & 1.683702 & 0.212924 & 3.567906 \\
$\mathrm{O}$ & 3.019295 & -1.849134 & 1.454516 \\
$\mathrm{~N}$ & -0.146682 & 2.309618 & 0.070700 \\
$\mathrm{C}$ & 0.298527 & 3.683737 & 0.273621 \\
$\mathrm{C}$ & 0.610742 & 4.124815 & 1.731389 \\
$\mathrm{O}$ & 1.092325 & 5.223485 & 1.887633 \\
$\mathrm{O}$ & 0.330837 & 3.310030 & 2.742807 \\
$\mathrm{H}$ & -1.041032 & -0.051751 & -0.324366 \\
$\mathrm{H}$ & 0.567620 & -0.836360 & -0.411096 \\
$\mathrm{H}$ & 2.597045 & 2.229173 & -0.506216 \\
$\mathrm{H}$ & 2.512771 & 0.489500 & -0.837015 \\
$\mathrm{H}$ & 2.424722 & 1.956344 & 1.845081 \\
$\mathrm{H}$ & 3.480258 & 0.622608 & 1.368907 \\
$\mathrm{H}$ & 0.811272 & -3.022783 & 2.778824 \\
$\mathrm{H}$ & 0.597080 & -2.998151 & 1.039959 \\
$\mathrm{H}$ & -0.817776 & -1.291984 & 3.134814 \\
$\mathrm{H}$ & -1.404241 & -1.689048 & 1.524473 \\
$\mathrm{H}$ & 1.133107 & -0.533931 & 4.148423 \\
$\mathrm{H}$ & 2.740398 & 0.143364 & 3.847972 \\
$\mathrm{H}$ & 1.312218 & 1.200671 & 3.852500 \\
$\mathrm{H}$ & -0.839857 & 1.851723 & 0.897494 \\
$\mathrm{H}$ & -0.085023 & 2.410053 & 2.505762 \\
$\mathrm{H}$ & -0.491886 & 4.352104 & -0.082864 \\
$\mathrm{H}$ & 1.191441 & 3.900577 & -0.317050
\end{tabular}

B3LYP / 6-31+G (d,p) / / B 3LYP/ 6-31G (d) =-823.1845231

\section{anti-9 - B3LYP/6-31+G(d,p) optimized}

\begin{tabular}{|c|c|c|c|}
\hline $\mathrm{SCF}$ & -823.1847928 & $/ / \mathrm{ZPE}=$ & 80038 \\
\hline $\mathrm{C}$ & -0.722550 & -1.797025 & -0.214089 \\
\hline $\mathrm{C}$ & -0.873985 & -0.349291 & -0.917429 \\
\hline $\mathrm{C}$ & -1.105806 & 0.675997 & 0.268945 \\
\hline $\mathrm{C}$ & -0.164056 & 0.550004 & 1.493578 \\
\hline $\mathrm{C}$ & 0.311784 & -0.903237 & 1.875547 \\
\hline $\mathrm{C}$ & 0.512648 & -1.569327 & 0.553872 \\
\hline $\mathrm{C}$ & -2.576466 & 0.398393 & 0.616598 \\
\hline $\mathrm{C}$ & -3.308933 & -0.110672 & -0.628896 \\
\hline $\mathrm{C}$ & -2.216965 & -0.334189 & -1.692721 \\
\hline O & 0.232031 & -0.156209 & -1.719447 \\
\hline $\mathrm{C}$ & -1.054308 & 2.141662 & -0.237718 \\
\hline O & -3.081299 & 0.547115 & 1.712208 \\
\hline $\mathrm{N}$ & 1.573224 & -1.484640 & -0.208633 \\
\hline $\mathrm{C}$ & 2.857611 & -0.923266 & 0.197671 \\
\hline $\mathrm{C}$ & 3.107562 & 0.570049 & -0.145469 \\
\hline O & 4.100857 & 1.091252 & 0.317347 \\
\hline $\mathrm{O}$ & 2.261485 & 1.213656 & -0.937800 \\
\hline $\mathrm{H}$ & -0.614429 & -2.536220 & -1.009478 \\
\hline $\mathrm{H}$ & -1.580162 & -2.038182 & 0.414895 \\
\hline $\mathrm{H}$ & 1.211664 & -0.840260 & 2.491070 \\
\hline $\mathrm{H}$ & -0.461826 & -1.422604 & 2.446577 \\
\hline $\mathrm{H}$ & 0.727176 & 1.154040 & 1.306831 \\
\hline $\mathrm{H}$ & -0.673559 & 0.979588 & 2.362482 \\
\hline $\mathrm{H}$ & -4.065895 & 0.622577 & -0.927887 \\
\hline $\mathrm{H}$ & -3.855287 & -1.021036 & -0.358738 \\
\hline $\mathrm{H}$ & -2.174914 & 0.489624 & -2.410373 \\
\hline $\mathrm{H}$ & -2.349887 & -1.254860 & -2.267788 \\
\hline $\mathrm{H}$ & -1.784802 & 2.330871 & -1.029248 \\
\hline $\mathrm{H}$ & -1.268224 & 2.827571 & 0.588120 \\
\hline $\mathrm{H}$ & -0.062734 & 2.370954 & -0.634473 \\
\hline $\mathrm{H}$ & 1.131793 & -1.100807 & -1.217610 \\
\hline $\mathrm{H}$ & 1.426657 & 0.706618 & -1.253443 \\
\hline $\mathrm{H}$ & 3.648538 & -1.484467 & -0.309551 \\
\hline $\mathrm{H}$ & 3.020467 & -1.035094 & 1.271212 \\
\hline
\end{tabular}




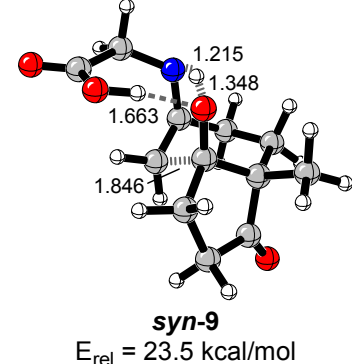
34

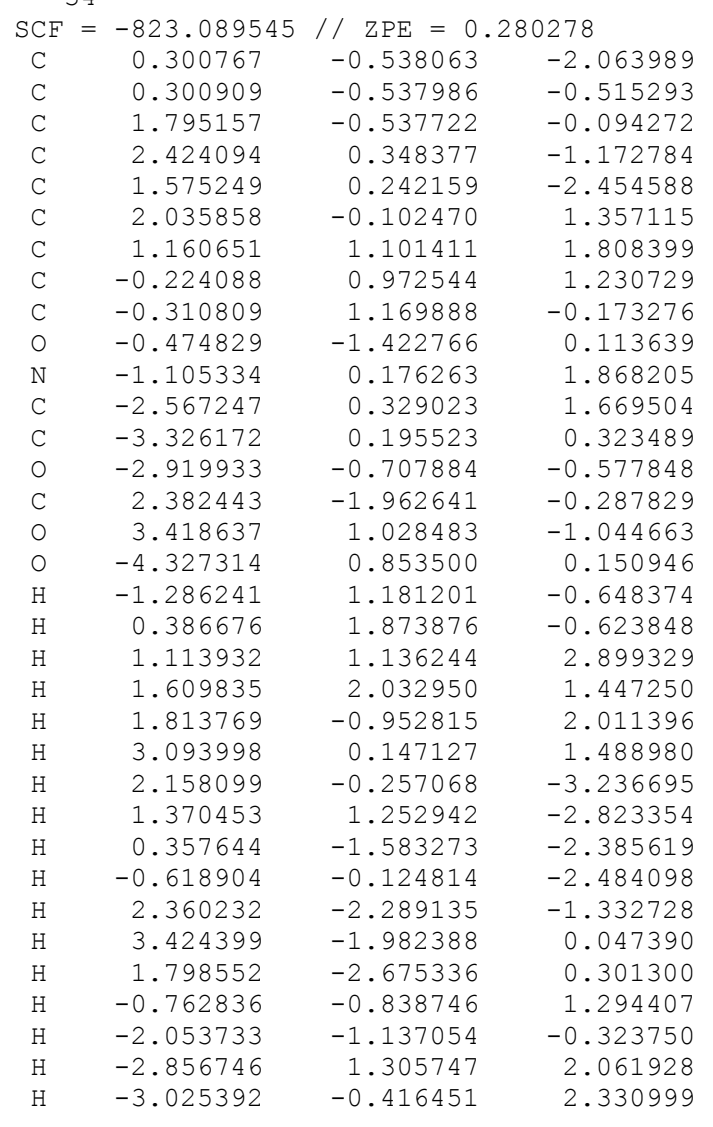

B3LYP / 6-31+G (d,p) / / B3LYP/6-31G (d) =-823.1528089

\section{syn-9 - B3LYP/6-31+G(d,p) optimized}

\begin{tabular}{|c|c|c|c|}
\hline $\mathrm{SCF}$ & -823.1530836 & $/ / \mathrm{ZPE}=$ & 78593 \\
\hline C & 0.807941 & -1.997858 & -0.034176 \\
\hline C & 0.455021 & -0.549707 & 0.377473 \\
\hline C & 1.806769 & 0.206383 & 0.482488 \\
\hline $\mathrm{C}$ & 2.609653 & -0.432628 & -0.653868 \\
\hline C & 2.085606 & -1.858545 & -0.891250 \\
\hline $\mathrm{C}$ & 1.670781 & 1.734393 & 0.449093 \\
\hline $\mathrm{C}$ & 0.639918 & 2.254990 & -0.592576 \\
\hline $\mathrm{C}$ & -0.560419 & 1.347343 & -0.626123 \\
\hline $\mathrm{C}$ & -0.338561 & 0.069994 & -1.200076 \\
\hline O & -0.395451 & -0.363197 & 1.386537 \\
\hline $\mathrm{N}$ & -1.521386 & 1.524630 & 0.304536 \\
\hline $\mathrm{C}$ & -2.903225 & 1.034595 & 0.077512 \\
\hline $\mathrm{C}$ & -3.331593 & -0.448163 & -0.056139 \\
\hline O & -2.685885 & -1.395361 & 0.632911 \\
\hline $\mathrm{C}$ & 2.521489 & -0.197895 & 1.803714 \\
\hline O & 3.514520 & 0.091474 & -1.270664 \\
\hline O & -4.309672 & -0.712640 & -0.723966 \\
\hline $\mathrm{H}$ & -1.175959 & -0.607791 & -1.330062 \\
\hline $\mathrm{H}$ & 0.399510 & -0.004360 & -1.995574 \\
\hline $\mathrm{H}$ & 0.336275 & 3.271622 & -0.334465 \\
\hline $\mathrm{H}$ & 1.100093 & 2.268172 & -1.586030 \\
\hline $\mathrm{H}$ & 1.354863 & 2.068358 & 1.443173 \\
\hline $\mathrm{H}$ & 2.649198 & 2.182061 & 0.248149 \\
\hline $\mathrm{H}$ & 2.864999 & -2.577967 & -0.618300 \\
\hline $\mathrm{H}$ & 1.907574 & -1.990950 & -1.963342 \\
\hline $\mathrm{H}$ & 1.002660 & -2.554784 & 0.888038 \\
\hline $\mathrm{H}$ & -0.012889 & -2.504343 & -0.545557 \\
\hline $\mathrm{H}$ & 2.754780 & -1.265842 & 1.846993 \\
\hline $\mathrm{H}$ & 3.460538 & 0.355749 & 1.895903 \\
\hline $\mathrm{H}$ & 1.874476 & 0.042034 & 2.651355 \\
\hline $\mathrm{H}$ & -0.993255 & 0.806834 & 1.129153 \\
\hline $\mathrm{H}$ & -1.845599 & -1.064952 & 1.060928 \\
\hline $\mathrm{H}$ & -3.295411 & 1.527587 & -0.813734 \\
\hline $\mathrm{H}$ & -3.488726 & 1.414896 & 0.922627 \\
\hline
\end{tabular}




\begin{tabular}{|c|c|c|c|}
\hline \multicolumn{4}{|c|}{ syn-b (Ph anti) } \\
\hline \multicolumn{4}{|c|}{0.0} \\
\hline & r & & \\
\hline $\mathrm{SCF}$ & -1093.515068 & $/ / \mathrm{ZPE}=$ & 92037 \\
\hline$C$ & 0.697193 & -1.364259 & -3.703564 \\
\hline $\mathrm{C}$ & 0.718144 & -1.455537 & -2.175261 \\
\hline $\mathrm{C}$ & 2.158142 & -1.597979 & -1.682609 \\
\hline C & 2.983841 & -1.035167 & -2.848143 \\
\hline $\mathrm{C}$ & 2.113570 & -0.914899 & -4.109629 \\
\hline 0 & -0.268390 & -1.403743 & -1.465305 \\
\hline $\mathrm{C}$ & 2.428338 & -0.918998 & -0.332163 \\
\hline $\mathrm{C}$ & 2.103332 & 0.602216 & -0.288792 \\
\hline C & 1.372150 & 0.988940 & 0.978130 \\
\hline $\mathrm{N}$ & 0.054389 & 0.526041 & 0.993608 \\
\hline $\mathrm{C}$ & -0.693877 & 0.440903 & 2.220065 \\
\hline $\mathrm{C}$ & -0.094068 & -0.587118 & 3.190730 \\
\hline 0 & -0.358851 & -0.271243 & 4.482868 \\
\hline C & 2.475640 & -3.119301 & -1.590147 \\
\hline 0 & 4.154588 & -0.733373 & -2.785485 \\
\hline $\mathrm{C}$ & 1.959079 & 1.704778 & 1.954493 \\
\hline 0 & 0.497271 & -1.593937 & 2.870450 \\
\hline $\mathrm{H}$ & 0.012099 & -0.995009 & 5.023018 \\
\hline $\mathrm{H}$ & 1.463572 & 1.963195 & 2.884278 \\
\hline $\mathrm{H}$ & 2.972182 & 2.067540 & 1.825727 \\
\hline $\mathrm{H}$ & 1.471165 & 0.882585 & -1.143580 \\
\hline $\mathrm{H}$ & 3.022771 & 1.185655 & -0.377916 \\
\hline $\mathrm{H}$ & 1.846033 & -1.445209 & 0.431909 \\
\hline $\mathrm{H}$ & 3.483462 & -1.072959 & -0.084071 \\
\hline $\mathrm{H}$ & 2.552753 & -1.506177 & -4.919931 \\
\hline $\mathrm{H}$ & 2.141821 & 0.130887 & -4.437556 \\
\hline $\mathrm{H}$ & 0.451687 & -2.356634 & -4.103940 \\
\hline $\mathrm{H}$ & -0.102705 & -0.689287 & -4.020393 \\
\hline $\mathrm{H}$ & 2.292473 & -3.645751 & -2.534102 \\
\hline $\mathrm{H}$ & 1.854902 & -3.577664 & -0.813769 \\
\hline $\mathrm{H}$ & 3.530445 & -3.249013 & -1.328801 \\
\hline $\mathrm{H}$ & -0.118717 & -0.248368 & 0.358837 \\
\hline $\mathrm{C}$ & -2.165377 & 0.032205 & 1.935102 \\
\hline $\mathrm{H}$ & -0.698133 & 1.417994 & 2.714276 \\
\hline $\mathrm{H}$ & -2.663525 & -0.117596 & 2.899707 \\
\hline C & -2.927999 & 1.047724 & 1.111783 \\
\hline $\mathrm{H}$ & -2.154018 & -0.940270 & 1.426984 \\
\hline C & -2.989782 & 0.947778 & -0.284566 \\
\hline $\mathrm{C}$ & -3.688612 & 1.894525 & -1.034550 \\
\hline C & -4.335849 & 2.956881 & -0.401005 \\
\hline C & -4.280913 & 3.065856 & 0.989467 \\
\hline $\mathrm{C}$ & -3.583744 & 2.116224 & 1.737131 \\
\hline $\mathrm{H}$ & -2.484540 & 0.126837 & -0.787615 \\
\hline $\mathrm{H}$ & -3.728884 & 1.800647 & -2.116886 \\
\hline $\mathrm{H}$ & -4.881971 & 3.692361 & -0.985894 \\
\hline $\mathrm{H}$ & -4.785689 & 3.886040 & 1.493519 \\
\hline $\mathrm{H}$ & -3.551751 & 2.202649 & 2.821590 \\
\hline
\end{tabular}

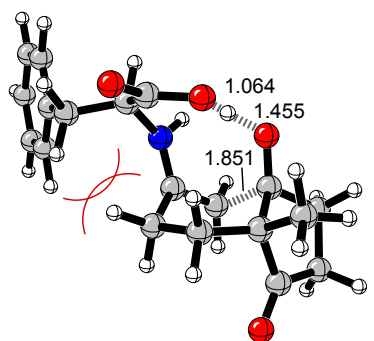

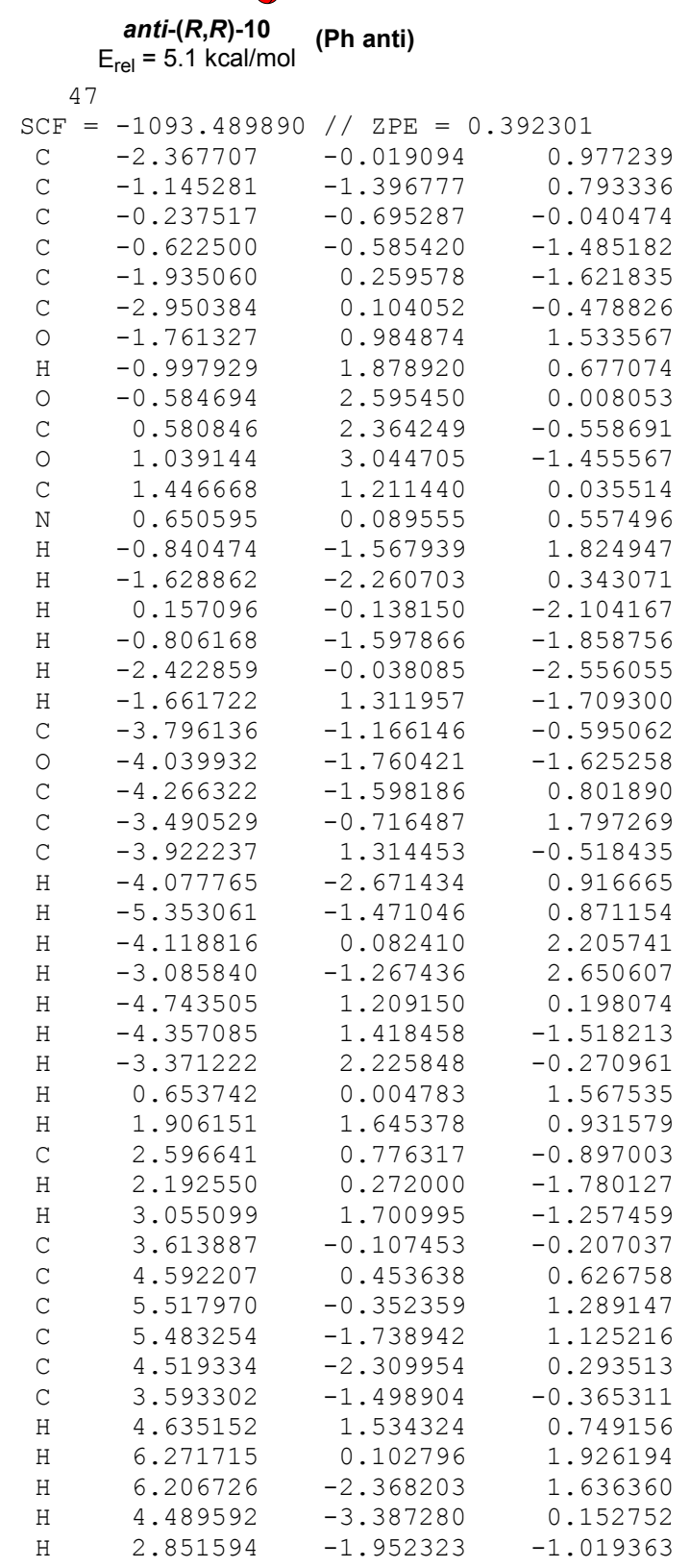




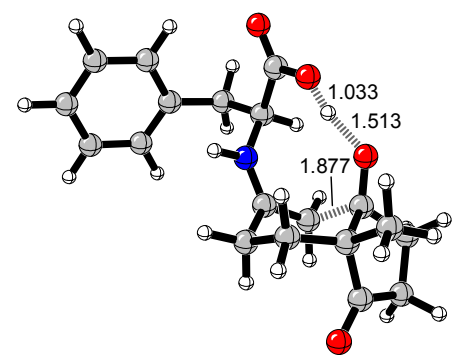

syn- $(\boldsymbol{R}, \boldsymbol{R})-10$
$\mathrm{E}_{\mathrm{rel}}=1.7 \mathrm{kcal} / \mathrm{mol}$$\quad$ (Ph gauche-in)

$$
\begin{aligned}
& \mathrm{SCF}=-1093.496069 / / \mathrm{ZPE}=0.393000
\end{aligned}
$$

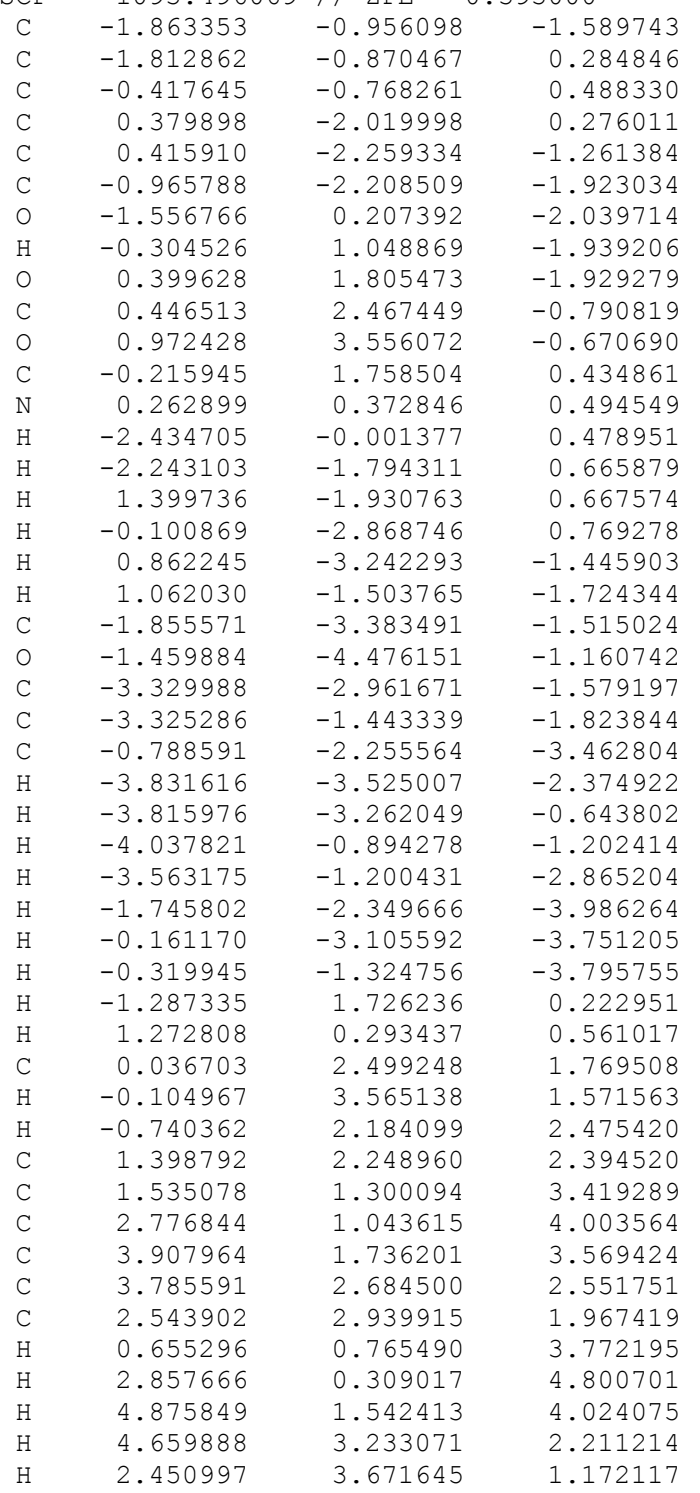

B3LYP / 6-31+G (d,p) / / B3LYP/6-31G (d)= $-1093.5781141$

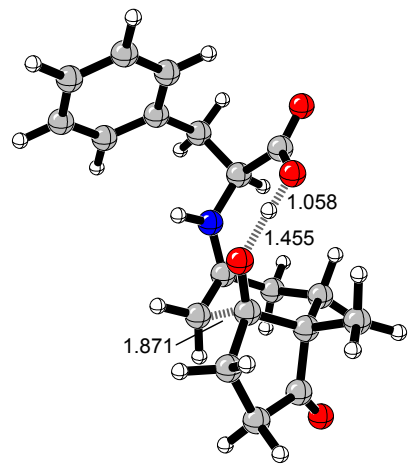

\begin{tabular}{|c|c|c|c|}
\hline & $\begin{array}{c}\text { anti-(S,S)-10 } \\
\mathrm{E}_{\text {rel }}=0.0 \mathrm{kcal} / \mathrm{mol} \\
\left(\mathrm{E}_{\mathrm{act}}=10.8 \mathrm{kcal} / \mathrm{mol}\right.\end{array}$ & (Ph gauch & he-in) \\
\hline & & & \\
\hline $\mathrm{SCF}$ & -1093.498374 & $/ / \mathrm{ZPE}=$ & 0.392629 \\
\hline $\mathrm{C}$ & -1.687130 & 1.035052 & -0.862266 \\
\hline $\mathrm{C}$ & -1.682233 & 0.996981 & 1.009135 \\
\hline $\mathrm{C}$ & -0.277384 & 1.062183 & 1.168164 \\
\hline $\mathrm{C}$ & 0.341420 & 2.415110 & 0.989479 \\
\hline $\mathrm{C}$ & 0.293340 & 2.755892 & -0.530855 \\
\hline $\mathrm{C}$ & -1.049515 & 2.432731 & -1.201768 \\
\hline 0 & -1.123005 & -0.056592 & -1.261775 \\
\hline $\mathrm{H}$ & 0.323795 & -0.165951 & -1.353546 \\
\hline O & 1.344339 & -0.289376 & -1.608154 \\
\hline $\mathrm{C}$ & 2.248069 & -0.440803 & -0.669797 \\
\hline 0 & 3.418688 & -0.687182 & -0.895549 \\
\hline $\mathrm{C}$ & 1.852076 & -0.246579 & 0.831836 \\
\hline $\mathrm{H}$ & 2.368662 & 0.663954 & 1.146011 \\
\hline $\mathrm{N}$ & 0.423840 & -0.061725 & 1.068579 \\
\hline $\mathrm{H}$ & -2.165848 & 0.039105 & 1.191313 \\
\hline $\mathrm{H}$ & -2.240031 & 1.845670 & 1.398645 \\
\hline $\mathrm{H}$ & 1.370484 & 2.474898 & 1.353839 \\
\hline $\mathrm{H}$ & -0.250807 & 3.147967 & 1.544365 \\
\hline $\mathrm{H}$ & 1.087554 & 2.208747 & -1.046667 \\
\hline $\mathrm{H}$ & 0.494480 & 3.826438 & -0.645574 \\
\hline $\mathrm{C}$ & -2.163097 & 3.404727 & -0.807469 \\
\hline 0 & -2.001398 & 4.551089 & -0.440795 \\
\hline $\mathrm{C}$ & -3.521949 & 2.696739 & -0.909808 \\
\hline $\mathrm{C}$ & -3.206114 & 1.209091 & -1.148013 \\
\hline $\mathrm{C}$ & -0.868891 & 2.519200 & -2.740818 \\
\hline $\mathrm{H}$ & -4.103643 & 3.148681 & -1.721848 \\
\hline $\mathrm{H}$ & -4.084432 & 2.890382 & 0.010487 \\
\hline $\mathrm{H}$ & -3.359394 & 0.925798 & -2.195231 \\
\hline $\mathrm{H}$ & -3.812904 & 0.528109 & -0.545357 \\
\hline $\mathrm{H}$ & -1.819081 & 2.414561 & -3.274801 \\
\hline $\mathrm{H}$ & -0.430105 & 3.483456 & -3.018573 \\
\hline $\mathrm{H}$ & -0.207626 & 1.713293 & -3.071564 \\
\hline $\mathrm{H}$ & -0.121852 & -0.919457 & 1.019806 \\
\hline $\mathrm{C}$ & 2.390371 & -1.416998 & 1.693514 \\
\hline $\mathrm{H}$ & 3.451854 & -1.507887 & 1.449843 \\
\hline $\mathrm{H}$ & 2.308668 & -1.129268 & 2.747795 \\
\hline $\mathrm{C}$ & 1.672264 & -2.731867 & 1.461171 \\
\hline $\mathrm{C}$ & 1.995763 & -3.545123 & 0.363283 \\
\hline $\mathrm{C}$ & 1.312826 & -4.740388 & 0.140722 \\
\hline $\mathrm{C}$ & 0.296495 & -5.145728 & 1.009047 \\
\hline $\mathrm{C}$ & -0.032051 & -4.348895 & 2.105683 \\
\hline $\mathrm{C}$ & 0.652314 & -3.151528 & 2.328303 \\
\hline $\mathrm{H}$ & 2.785435 & -3.235449 & -0.315253 \\
\hline $\mathrm{H}$ & 1.577645 & -5.358060 & -0.713113 \\
\hline $\mathrm{H}$ & -0.232046 & -6.078760 & 0.833782 \\
\hline $\mathrm{H}$ & -0.814749 & -4.659559 & 2.792795 \\
\hline $\mathrm{H}$ & 0.405211 & -2.544258 & 3.197364 \\
\hline
\end{tabular}

B3LYP / 6-31+G (d,p) / /B3LYP / 6-31G $(d)=$ $-1093.5802753$ 


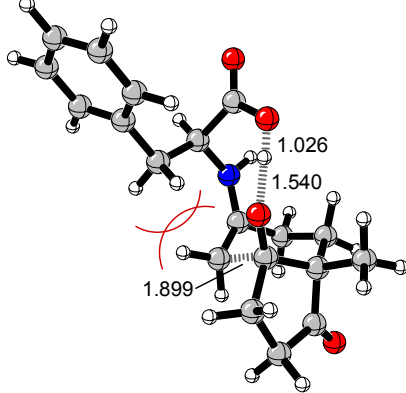

syn-(S,S)-10

$\mathrm{E}_{\mathrm{rel}}=8.7 \mathrm{kcal} / \mathrm{mol}$

\begin{tabular}{|c|c|c|c|}
\hline $\mathrm{SCF}$ & $=-1093.485446$ & $/ / \mathrm{ZPE}=$ & 3501 \\
\hline $\mathrm{C}$ & 1.840475 & -0.924245 & 0.259274 \\
\hline $\mathrm{C}$ & 1.346855 & -0.361108 & -1.486191 \\
\hline $\mathrm{C}$ & 1.149984 & 1.020260 & -1.268498 \\
\hline $\mathrm{C}$ & 2.410963 & 1.821892 & -1.090730 \\
\hline $\mathrm{C}$ & 2.952477 & 1.476938 & 0.325721 \\
\hline $\mathrm{C}$ & 3.096920 & -0.028640 & 0.577011 \\
\hline 0 & 0.794889 & -0.873226 & 1.002448 \\
\hline $\mathrm{H}$ & -0.083554 & 0.306500 & 1.457702 \\
\hline O & -0.736101 & 0.990737 & 1.856582 \\
\hline $\mathrm{C}$ & -1.529647 & 1.594499 & 0.985681 \\
\hline O & -2.343995 & 2.436889 & 1.296369 \\
\hline $\mathrm{C}$ & -1.345197 & 1.215299 & -0.517156 \\
\hline $\mathrm{N}$ & 0.030688 & 1.633773 & -0.882615 \\
\hline $\mathrm{H}$ & 0.498730 & -1.000213 & -1.688677 \\
\hline $\mathrm{H}$ & 2.203796 & -0.574171 & -2.121522 \\
\hline $\mathrm{H}$ & 2.232831 & 2.900843 & -1.171272 \\
\hline $\mathrm{H}$ & 3.151370 & 1.546688 & -1.846049 \\
\hline $\mathrm{H}$ & 3.932755 & 1.951240 & 0.443357 \\
\hline $\mathrm{H}$ & 2.280381 & 1.903274 & 1.080514 \\
\hline C & 4.193507 & -0.658045 & -0.285127 \\
\hline 0 & 5.175927 & -0.078835 & -0.703322 \\
\hline C & 3.849734 & -2.124820 & -0.575986 \\
\hline $\mathrm{C}$ & 2.421148 & -2.336509 & -0.049253 \\
\hline C & 3.468327 & -0.258829 & 2.064953 \\
\hline $\mathrm{H}$ & 4.589450 & -2.771350 & -0.089255 \\
\hline $\mathrm{H}$ & 3.958624 & -2.298074 & -1.652814 \\
\hline $\mathrm{H}$ & 2.418068 & -2.879206 & 0.902533 \\
\hline $\mathrm{H}$ & 1.777112 & -2.896838 & -0.732229 \\
\hline $\mathrm{H}$ & 3.690412 & -1.310192 & 2.275606 \\
\hline $\mathrm{H}$ & 4.347673 & 0.334665 & 2.336341 \\
\hline $\mathrm{H}$ & 2.621933 & 0.033272 & 2.693460 \\
\hline $\mathrm{H}$ & 0.166123 & 2.614030 & -0.652782 \\
\hline $\mathrm{H}$ & -2.018718 & 1.886134 & -1.057825 \\
\hline C & -1.738673 & -0.240145 & -0.829444 \\
\hline $\mathrm{H}$ & -1.101243 & -0.916882 & -0.252776 \\
\hline $\mathrm{H}$ & -1.564685 & -0.425431 & -1.895522 \\
\hline $\mathrm{C}$ & -3.199268 & -0.499376 & -0.510205 \\
\hline $\mathrm{C}$ & -4.190691 & -0.299505 & -1.479058 \\
\hline C & -5.533630 & -0.537675 & -1.185811 \\
\hline $\mathrm{C}$ & -5.902805 & -0.980490 & 0.085535 \\
\hline $\mathrm{C}$ & -4.922706 & -1.182901 & 1.058464 \\
\hline $\mathrm{C}$ & -3.580279 & -0.945355 & 0.762115 \\
\hline $\mathrm{H}$ & -3.908383 & 0.038122 & -2.474607 \\
\hline $\mathrm{H}$ & -6.289897 & -0.381212 & -1.950557 \\
\hline $\mathrm{H}$ & -6.948088 & -1.168238 & 0.315799 \\
\hline $\mathrm{H}$ & -5.201543 & -1.527687 & 2.050476 \\
\hline $\mathrm{H}$ & -2.820732 & -1.101286 & $1.52403^{\circ}$ \\
\hline
\end{tabular}

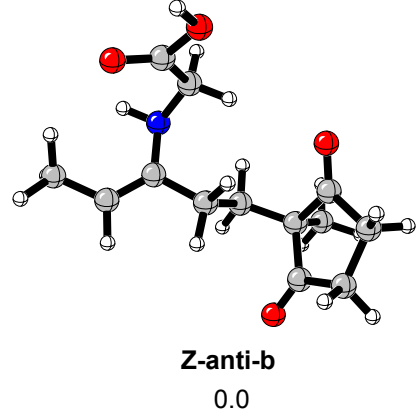

37

\begin{tabular}{|c|c|c|c|}
\hline $\mathrm{SCF}$ & -862.46264 & $/ / \mathrm{ZPE}=0$ & 0182 \\
\hline C & -3.396694 & -1.418985 & -1.376144 \\
\hline C & -3.428374 & -1.443975 & 0.162050 \\
\hline C & -1.960779 & -1.472556 & 0.606505 \\
\hline C & -1.078358 & -1.966893 & -0.543744 \\
\hline C & -1.955544 & -1.760745 & -1.786062 \\
\hline O & -1.563399 & -1.148736 & 1.706584 \\
\hline $\mathrm{C}$ & 0.288849 & -1.266543 & -0.618916 \\
\hline $\mathrm{C}$ & 0.216757 & 0.262116 & -0.804045 \\
\hline $\mathrm{C}$ & 1.559507 & 0.943852 & -0.651231 \\
\hline C & 2.127950 & 1.615881 & -1.669590 \\
\hline $\mathrm{C}$ & -0.880826 & -3.498847 & -0.364226 \\
\hline O & -1.569769 & -1.857258 & -2.930381 \\
\hline $\mathrm{N}$ & 2.220052 & 0.732346 & 0.584328 \\
\hline $\mathrm{C}$ & 1.525886 & 0.979561 & 1.834760 \\
\hline $\mathrm{C}$ & 1.395458 & 2.459124 & 2.187520 \\
\hline O & 2.040580 & 3.356861 & 1.693023 \\
\hline O & 0.492902 & 2.658725 & 3.176997 \\
\hline $\mathrm{H}$ & 0.508035 & 3.614994 & 3.373026 \\
\hline C & 3.433820 & 2.363385 & -1.612550 \\
\hline $\mathrm{H}$ & 1.610286 & 1.599861 & -2.625559 \\
\hline $\mathrm{H}$ & -0.499345 & 0.682476 & -0.083256 \\
\hline $\mathrm{H}$ & -0.175139 & 0.497554 & -1.798734 \\
\hline $\mathrm{H}$ & 0.838156 & -1.499398 & 0.298708 \\
\hline $\mathrm{H}$ & 0.852922 & -1.699688 & -1.452626 \\
\hline $\mathrm{H}$ & -4.093705 & -2.116353 & -1.852032 \\
\hline $\mathrm{H}$ & -3.628171 & -0.425263 & -1.779059 \\
\hline $\mathrm{H}$ & -3.917715 & -2.349255 & 0.545085 \\
\hline $\mathrm{H}$ & -3.936776 & -0.592250 & 0.622381 \\
\hline $\mathrm{H}$ & -1.832848 & -4.037930 & -0.293162 \\
\hline $\mathrm{H}$ & -0.309771 & -3.690406 & 0.549790 \\
\hline $\mathrm{H}$ & -0.330508 & -3.893823 & -1.223747 \\
\hline $\mathrm{H}$ & 3.131621 & 1.177241 & 0.593636 \\
\hline $\mathrm{H}$ & 2.085032 & 0.509344 & 2.655614 \\
\hline $\mathrm{H}$ & 0.540339 & 0.507381 & 1.842866 \\
\hline $\mathrm{H}$ & 3.453991 & 3.159741 & -2.364659 \\
\hline $\mathrm{H}$ & 3.584890 & 2.839665 & -0.635597 \\
\hline $\mathrm{H}$ & 4.303796 & 1.719089 & -1.812285 \\
\hline
\end{tabular}




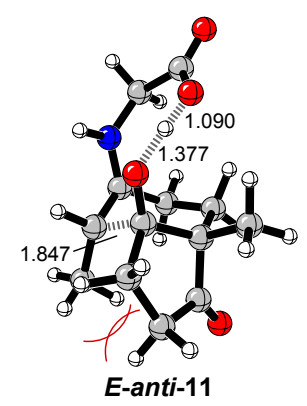

$\mathrm{E}_{\mathrm{rel}}=4.6 \mathrm{kcal} / \mathrm{mo}$

37

\begin{tabular}{crrr}
$\mathrm{SCF}$ & -162.436272 & \multicolumn{1}{c}{$/ \mathrm{ZPE}=0.310341$} \\
$\mathrm{C}$ & 0.751062 & -0.147404 & -0.981612 \\
$\mathrm{C}$ & 0.616430 & 1.660019 & -0.627072 \\
$\mathrm{C}$ & -0.523499 & 1.539185 & 0.232022 \\
$\mathrm{C}$ & -0.293641 & 1.068245 & 1.631832 \\
$\mathrm{C}$ & 0.022194 & -0.459332 & 1.555426 \\
$\mathrm{C}$ & 0.959021 & -0.865312 & 0.404730 \\
$\mathrm{O}$ & -0.299010 & -0.445013 & -1.702975 \\
$\mathrm{H}$ & -1.465676 & -0.844126 & -1.089704 \\
$\mathrm{O}$ & -2.375552 & -1.295359 & -0.692664 \\
$\mathrm{C}$ & -3.267244 & -0.569257 & -0.069321 \\
$\mathrm{O}$ & -4.333962 & -0.974745 & 0.347308 \\
$\mathrm{C}$ & -2.963774 & 0.936482 & 0.204719 \\
$\mathrm{~N}$ & -1.719650 & 1.471607 & -0.337802 \\
$\mathrm{H}$ & -1.151446 & 1.238236 & 2.287455 \\
$\mathrm{H}$ & 0.557089 & 1.601129 & 2.060730 \\
$\mathrm{H}$ & -0.917681 & -1.008143 & 1.454411 \\
$\mathrm{H}$ & 0.483609 & -0.759685 & 2.502361 \\
$\mathrm{C}$ & 2.433952 & -0.596832 & 0.704247 \\
$\mathrm{O}$ & 2.922413 & -0.488915 & 1.811081 \\
$\mathrm{C}$ & 3.198363 & -0.466300 & -0.617607 \\
$\mathrm{C}$ & 2.125251 & -0.277210 & -1.708775 \\
$\mathrm{C}$ & 0.825381 & -2.398610 & 0.183433 \\
$\mathrm{H}$ & 3.792341 & -1.375886 & -0.769807 \\
$\mathrm{H}$ & 3.914379 & 0.356732 & -0.535077 \\
$\mathrm{H}$ & 2.041857 & -1.156446 & -2.354695 \\
$\mathrm{H}$ & 2.321832 & 0.575006 & -2.365848 \\
$\mathrm{H}$ & 1.533280 & -2.770842 & -0.563951 \\
$\mathrm{H}$ & -0.183964 & -2.629860 & -0.165586 \\
$\mathrm{H}$ & 1.011300 & -2.931443 & 1.122326 \\
$\mathrm{H}$ & -2.986152 & 1.079015 & 1.285934 \\
$\mathrm{H}$ & -3.793827 & 1.513777 & -0.210341 \\
$\mathrm{H}$ & -1.711449 & 1.596266 & -1.346229 \\
$\mathrm{H}$ & 0.350334 & 1.981028 & -1.637001 \\
$\mathrm{C}$ & 1.852544 & 2.409101 & -0.133921 \\
$\mathrm{H}$ & 2.650586 & 2.348658 & -0.878871 \\
$\mathrm{H}$ & 2.254186 & 2.036105 & 0.810908 \\
$\mathrm{H}$ & 1.624394 & 3.474144 & -0.000315 \\
& & &
\end{tabular}

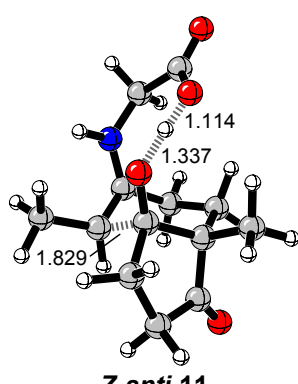

Z-anti-11

$E_{\text {rel }}=0.0 \mathrm{kcal} / \mathrm{mol}$

$\left(E_{\text {act }}=12.1 \mathrm{kcal} / \mathrm{mol}\right)$

37

$\mathrm{SCF}=-862.442879 / / \mathrm{ZPE}=0.309669$

$\begin{array}{rrrr}\mathrm{C} & 0.845041 & 0.347691 & -0.809998 \\ \mathrm{C} & 0.656117 & 1.624480 & 0.485521 \\ \mathrm{C} & -0.494527 & 1.033830 & 1.103900 \\ \mathrm{C} & -0.252579 & -0.141235 & 1.995409 \\ \mathrm{C} & 0.112069 & -1.352699 & 1.088071 \\ \mathrm{C} & 1.096170 & -1.001746 & -0.036491 \\ \mathrm{O} & -0.184998 & 0.450418 & -1.615335 \\ \mathrm{H} & -1.317861 & -0.192348 & -1.312574 \\ \mathrm{O} & -2.242893 & -0.805909 & -1.222320 \\ \mathrm{C} & -3.177278 & -0.548263 & -0.349319 \\ \mathrm{O} & -4.249556 & -1.116521 & -0.271679 \\ \mathrm{C} & -2.927346 & 0.550373 & 0.734664 \\ \mathrm{~N} & -1.690483 & 1.314707 & 0.612176 \\ \mathrm{H} & -1.111992 & -0.387057 & 2.624677 \\ \mathrm{H} & 0.590162 & 0.086751 & 2.654148 \\ \mathrm{H} & -0.801498 & -1.759801 & 0.647999 \\ \mathrm{H} & 0.559588 & -2.129029 & 1.717618 \\ \mathrm{C} & 2.530787 & -0.825385 & 0.469686 \\ \mathrm{O} & 2.991904 & -1.335670 & 1.470078 \\ \mathrm{C} & 3.287612 & 0.127401 & -0.465286 \\ \mathrm{C} & 2.231019 & 0.680216 & -1.436230 \\ \mathrm{C} & 1.102729 & -2.168478 & -1.061288 \\ \mathrm{H} & 4.095830 & -0.417343 & -0.966871 \\ \mathrm{H} & 3.773659 & 0.897336 & 0.145470 \\ \mathrm{H} & 2.260335 & 0.162740 & -2.400628 \\ \mathrm{H} & 2.346546 & 1.744026 & -1.652786 \\ \mathrm{H} & 1.873504 & -2.045006 & -1.828598 \\ \mathrm{H} & 0.132123 & -2.215294 & -1.562411 \\ \mathrm{H} & 1.288479 & -3.117622 & -0.547511 \\ \mathrm{H} & -2.964360 & 0.059252 & 1.708238 \\ \mathrm{H} & -3.767337 & 1.247602 & 0.693917 \\ \mathrm{H} & -1.696982 & 2.015774 & -0.121686 \\ \mathrm{C} & 0.504937 & 2.973276 & -0.207025 \\ \mathrm{H} & 1.532446 & 1.566853 & 1.131629 \\ \mathrm{H} & 1.483786 & 3.416224 & -0.406852 \\ \mathrm{H} & -0.008037 & 2.858205 & -1.169287 \\ \mathrm{H} & -0.049772 & 3.689253 & 0.412833\end{array}$ 


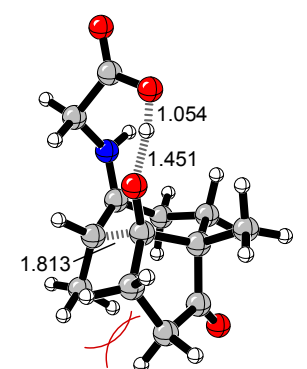

E-syn-11

$\mathrm{E}_{\mathrm{rel}}=7.9 \mathrm{kcal} / \mathrm{mo}$

37

\begin{tabular}{lrrr}
$\mathrm{SCF}$ & -162.432245 & \multicolumn{1}{c}{$/ \mathrm{ZPE}=0.311632$} \\
$\mathrm{C}$ & 0.590737 & -0.442882 & -0.810041 \\
$\mathrm{C}$ & 0.183229 & 1.323480 & -0.832638 \\
$\mathrm{C}$ & -0.554104 & 1.411254 & 0.397462 \\
$\mathrm{C}$ & 0.215245 & 1.345127 & 1.680823 \\
$\mathrm{C}$ & 0.625737 & -0.149708 & 1.833196 \\
$\mathrm{C}$ & 1.286026 & -0.742696 & 0.582631 \\
$\mathrm{O}$ & -0.504793 & -1.079237 & -1.117010 \\
$\mathrm{H}$ & -1.708552 & -1.322698 & -0.344715 \\
$\mathrm{O}$ & -2.659748 & -1.521011 & 0.062897 \\
$\mathrm{C}$ & -3.498823 & -0.519574 & -0.093711 \\
$\mathrm{O}$ & -4.692996 & -0.570426 & 0.116532 \\
$\mathrm{C}$ & -2.847447 & 0.827174 & -0.517945 \\
$\mathrm{~N}$ & -1.859673 & 1.210467 & 0.497343 \\
$\mathrm{H}$ & -0.391390 & 1.657438 & 2.538834 \\
$\mathrm{H}$ & 1.104412 & 1.976124 & 1.651616 \\
$\mathrm{H}$ & -0.270080 & -0.733609 & 2.076016 \\
$\mathrm{H}$ & 1.320545 & -0.230238 & 2.676097 \\
$\mathrm{C}$ & 2.710554 & -0.234447 & 0.371969 \\
$\mathrm{O}$ & 3.427467 & 0.236374 & 1.233692 \\
$\mathrm{C}$ & 3.078641 & -0.366314 & -1.109205 \\
$\mathrm{C}$ & 1.748889 & -0.602327 & -1.851372 \\
$\mathrm{C}$ & 1.359577 & -2.283281 & 0.746047 \\
$\mathrm{H}$ & 3.776699 & -1.204752 & -1.225319 \\
$\mathrm{H}$ & 3.623338 & 0.530695 & -1.419416 \\
$\mathrm{H}$ & 1.666378 & -1.628206 & -2.222028 \\
$\mathrm{H}$ & 1.612123 & 0.052148 & -2.717093 \\
$\mathrm{H}$ & 1.932258 & -2.755999 & -0.057945 \\
$\mathrm{H}$ & 0.346717 & -2.694105 & 0.713425 \\
$\mathrm{H}$ & 1.831203 & -2.545723 & 1.699340 \\
$\mathrm{H}$ & -0.451332 & 1.336943 & -1.718122 \\
$\mathrm{C}$ & 1.334185 & 2.323373 & -1.007983 \\
$\mathrm{H}$ & 0.948025 & 3.349263 & -1.052908 \\
$\mathrm{H}$ & 2.083114 & 2.286546 & -0.213899 \\
$\mathrm{H}$ & 1.846818 & 2.136033 & -1.954849 \\
$\mathrm{H}$ & -2.351265 & 0.713717 & -1.480840 \\
$\mathrm{H}$ & -3.633452 & 1.583496 & -0.571574 \\
$\mathrm{H}$ & -2.224500 & 1.169249 & 1.445182
\end{tabular}

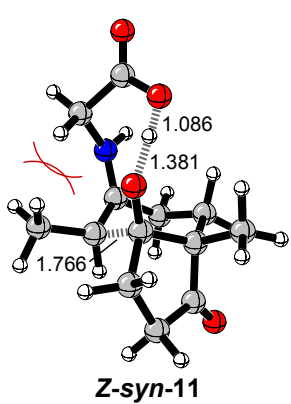

$\mathrm{E}_{\mathrm{rel}}=5.2 \mathrm{kcal} / \mathrm{mol}$ 37

\begin{tabular}{crrr}
$\mathrm{SCF}$ & -162.435655 & \multicolumn{1}{c}{$/ \mathrm{ZPE}=0.310795$} \\
$\mathrm{C}$ & -0.076799 & -0.312528 & -2.636120 \\
$\mathrm{C}$ & -0.088246 & -0.333186 & -1.072667 \\
$\mathrm{C}$ & 1.452063 & -0.327730 & -0.696896 \\
$\mathrm{C}$ & 2.075958 & 0.508140 & -1.818996 \\
$\mathrm{C}$ & 1.191640 & 0.438541 & -3.068276 \\
$\mathrm{C}$ & 1.827508 & 0.215657 & 0.686867 \\
$\mathrm{C}$ & 1.151743 & 1.581422 & 1.014309 \\
$\mathrm{C}$ & -0.307331 & 1.353070 & 0.764963 \\
$\mathrm{C}$ & -0.697373 & 1.262484 & -0.621866 \\
$\mathrm{O}$ & -0.873405 & -1.250531 & -0.556629 \\
$\mathrm{C}$ & -2.165043 & 1.388047 & -1.035004 \\
$\mathrm{~N}$ & -1.022468 & 0.974897 & 1.811994 \\
$\mathrm{C}$ & -2.225959 & 0.135481 & 1.905651 \\
$\mathrm{C}$ & -1.799474 & -1.226126 & 2.535640 \\
$\mathrm{O}$ & -2.181543 & -1.506860 & 3.654944 \\
$\mathrm{C}$ & 1.987719 & -1.776103 & -0.826808 \\
$\mathrm{O}$ & 3.097725 & 1.158410 & -1.722464 \\
$\mathrm{O}$ & -0.982378 & -1.944726 & 1.805178 \\
$\mathrm{H}$ & -0.885950 & -1.605844 & 0.778319 \\
$\mathrm{H}$ & 1.357499 & 1.862052 & 2.053875 \\
$\mathrm{H}$ & 1.551013 & 2.361709 & 0.362089 \\
$\mathrm{H}$ & 1.542279 & -0.514296 & 1.453232 \\
$\mathrm{H}$ & 2.912988 & 0.354065 & 0.733127 \\
$\mathrm{H}$ & 1.739488 & -0.060910 & -3.876023 \\
$\mathrm{H}$ & 1.006762 & 1.461937 & -3.416728 \\
$\mathrm{H}$ & -0.048674 & -1.361827 & -2.945688 \\
$\mathrm{H}$ & -0.996993 & 0.108406 & -3.047833 \\
$\mathrm{H}$ & 1.854149 & -2.175526 & -1.836957 \\
$\mathrm{H}$ & 1.438088 & -2.425206 & -0.139304 \\
$\mathrm{H}$ & 3.055748 & -1.811102 & -0.586829 \\
$\mathrm{H}$ & -2.949710 & 0.611721 & 2.569123 \\
$\mathrm{H}$ & -2.642745 & -0.018070 & 0.914570 \\
$\mathrm{H}$ & -0.542406 & 1.052836 & 2.705330 \\
$\mathrm{H}$ & -0.073195 & 1.965337 & -1.178646 \\
$\mathrm{H}$ & -2.233692 & 1.863142 & -2.017787 \\
$\mathrm{H}$ & -2.632739 & 0.401476 & -1.105841 \\
$\mathrm{H}$ & -2.742087 & 2.006994 & -0.339148
\end{tabular}




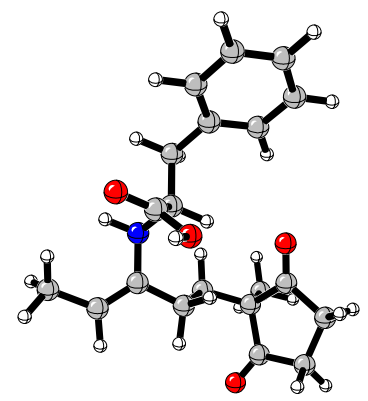

Z-anti-b (Ph gauche-out)

0.0

\begin{tabular}{lrrr}
$\mathrm{SCF}$ & -1132.829613 & \multicolumn{1}{l}{$/ /$ ZPE $=0.420084$} \\
$\mathrm{C}$ & -4.189790 & -0.746137 & 0.340997 \\
$\mathrm{C}$ & -4.141320 & -1.772287 & 1.483877 \\
$\mathrm{C}$ & -2.910210 & -2.657204 & 1.223332 \\
$\mathrm{C}$ & -2.108496 & -1.947197 & 0.126145 \\
$\mathrm{C}$ & -2.999858 & -0.940526 & -0.607719 \\
$\mathrm{O}$ & -0.941271 & -2.163568 & -0.127602 \\
$\mathrm{C}$ & -2.270915 & 0.362952 & -0.976627 \\
$\mathrm{C}$ & -1.788021 & 1.193974 & 0.229534 \\
$\mathrm{C}$ & -0.855440 & 2.318186 & -0.169091 \\
$\mathrm{~N}$ & 0.330274 & 1.918655 & -0.833402 \\
$\mathrm{C}$ & 1.219471 & 0.922691 & -0.221768 \\
$\mathrm{C}$ & 1.924663 & 1.538680 & 0.989163 \\
$\mathrm{O}$ & 1.547464 & 0.977215 & 2.159393 \\
$\mathrm{C}$ & -3.532667 & -1.633305 & -1.893283 \\
$\mathrm{O}$ & -5.051301 & 0.095286 & 0.207868 \\
$\mathrm{C}$ & -1.191267 & 3.610230 & 0.008655 \\
$\mathrm{C}$ & -0.341971 & 4.809431 & -0.318863 \\
$\mathrm{O}$ & 2.702309 & 2.468495 & 0.925461 \\
$\mathrm{H}$ & 2.023643 & 1.461513 & 2.861918 \\
$\mathrm{H}$ & -2.186414 & 3.812781 & 0.397859 \\
$\mathrm{H}$ & -1.285982 & 0.538269 & 0.955455 \\
$\mathrm{H}$ & -2.648211 & 1.626907 & 0.749584 \\
$\mathrm{H}$ & -1.414959 & 0.097992 & -1.605540 \\
$\mathrm{H}$ & -2.943437 & 0.978310 & -1.584738 \\
$\mathrm{H}$ & -5.087526 & -2.321198 & 1.528858 \\
$\mathrm{H}$ & -4.060664 & -1.215594 & 2.425626 \\
$\mathrm{H}$ & -3.191604 & -3.650102 & 0.848233 \\
$\mathrm{H}$ & -2.273036 & -2.820803 & 2.097053 \\
$\mathrm{H}$ & -4.037037 & -2.582531 & -1.677205 \\
$\mathrm{H}$ & -2.699167 & -1.836186 & -2.573233 \\
$\mathrm{H}$ & -4.250637 & -0.971644 & -2.387490 \\
$\mathrm{H}$ & 0.874367 & 2.718688 & -1.143300 \\
$\mathrm{C}$ & 2.257602 & 0.450319 & -1.259117 \\
$\mathrm{H}$ & 0.626741 & 0.066861 & 0.103109 \\
$\mathrm{H}$ & -0.547919 & 5.627622 & 0.380913 \\
$\mathrm{H}$ & 0.731473 & 4.593298 & -0.249307 \\
$\mathrm{H}$ & -0.537125 & 5.202233 & -1.328072 \\
$\mathrm{H}$ & 2.855260 & 1.315921 & -1.568453 \\
$\mathrm{C}$ & 3.165365 & -0.647024 & -0.742351 \\
$\mathrm{H}$ & 1.695255 & 0.108328 & -2.135195 \\
$\mathrm{C}$ & 2.685720 & -1.957955 & -0.601539 \\
$\mathrm{C}$ & 3.514470 & -2.968841 & -0.115333 \\
$\mathrm{C}$ & 4.836033 & -2.686017 & 0.238375 \\
$\mathrm{C}$ & 5.321220 & -1.384550 & 0.107257 \\
$\mathrm{C}$ & 4.489883 & -0.373207 & -0.378615 \\
$\mathrm{H}$ & 1.655028 & -2.183991 & -0.864978 \\
$\mathrm{H}$ & 3.127611 & -3.979627 & -0.014083 \\
$\mathrm{H}$ & 5.482331 & -3.475312 & 0.613595 \\
$\mathrm{H}$ & 4.869798 & -1.154242 & 0.380740 \\
& & 0.641023 & -0.474887
\end{tabular}

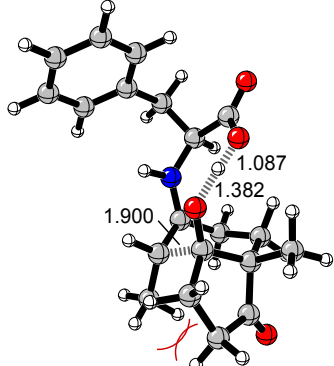

anti-(S,S,S)-12 $\mathrm{E}_{\mathrm{rel}}=4.9 \mathrm{kcal} / \mathrm{mol}$ (Ph gauche-in) 50

$\mathrm{SCF}=-1132.8055681 / / \mathrm{ZPE}=0.420531$

$\begin{array}{lrrr}\mathrm{C} & 0.000000 & 0.000000 & 0.000000 \\ \mathrm{C} & 0.000000 & 0.000000 & 1.404225 \\ \mathrm{C} & 1.232632 & 0.000000 & 2.074270 \\ \mathrm{C} & 2.435916 & -0.003514 & 1.364437 \\ \mathrm{C} & 2.421942 & -0.006180 & -0.030293 \\ \mathrm{C} & 1.200881 & -0.002962 & -0.708850 \\ \mathrm{C} & -1.301854 & -0.023382 & 2.181117 \\ \mathrm{C} & -1.930845 & -1.433452 & 2.312826 \\ \mathrm{~N} & -0.979787 & -2.351410 & 2.934094 \\ \mathrm{C} & -1.180348 & -3.304752 & 3.836482 \\ \mathrm{C} & -0.173388 & -4.305140 & 3.984347 \\ \mathrm{C} & -0.815972 & -5.367028 & 2.546066 \\ \mathrm{C} & -2.296879 & -5.748552 & 2.911940 \\ \mathrm{C} & -3.220115 & -4.618959 & 3.395766 \\ \mathrm{C} & -2.546696 & -3.605103 & 4.370040 \\ \mathrm{C} & -2.112047 & -6.858985 & 3.946102 \\ \mathrm{C} & -0.794813 & -7.591592 & 3.668221 \\ \mathrm{C} & -0.004264 & -6.687294 & 2.701640 \\ \mathrm{O} & -0.575811 & -4.670493 & 1.475958 \\ \mathrm{C} & 0.054575 & -4.972576 & 5.336271 \\ \mathrm{C} & -2.944578 & -6.407174 & 1.660710 \\ \mathrm{O} & -2.877917 & -7.114133 & 4.853753 \\ \mathrm{C} & -2.499468 & -1.915613 & 0.935152 \\ \mathrm{O} & -3.215971 & -1.128227 & 0.341465 \\ \mathrm{O} & -2.229500 & -3.123299 & 0.515840 \\ \mathrm{H} & -1.500080 & -3.746147 & 1.026722 \\ \mathrm{H} & -3.171592 & -2.713325 & 4.468077 \\ \mathrm{H} & -2.459603 & -4.050257 & 5.363006 \\ \mathrm{H} & -3.599339 & -4.075761 & 2.525842 \\ \mathrm{H} & -4.078156 & -5.068099 & 3.907536 \\ \mathrm{H} & -1.024744 & -8.570769 & 3.230303 \\ \mathrm{H} & -0.291969 & -7.791189 & 4.619119 \\ \mathrm{H} & 1.022904 & -6.498871 & 3.026594 \\ \mathrm{H} & 0.060971 & -7.121638 & 1.699059 \\ \mathrm{H} & -2.382977 & -7.281966 & 1.317589 \\ \mathrm{H} & -2.971474 & -5.683304 & 0.842156 \\ \mathrm{H} & -3.966387 & -6.728217 & 1.890609 \\ \mathrm{H} & 0.773824 & -4.029139 & 3.516092 \\ \mathrm{H} & 0.477310 & -4.255287 & 6.051819 \\ \mathrm{H} & 0.781304 & -5.783739 & 5.237937 \\ \mathrm{H} & -0.848759 & -5.392550 & 5.784616 \\ \mathrm{H} & -0.052598 & -2.331501 & 2.515251 \\ \mathrm{H} & -2.801600 & -1.350191 & 2.967888 \\ \mathrm{H} & -1.141357 & 0.376504 & 3.188902 \\ \mathrm{H} & -2.056298 & 0.594703 & 1.688232 \\ \mathrm{H} & 1.249843 & 0.022835 & 3.162495 \\ \mathrm{H} & 3.380527 & 0.002819 & 1.902021 \\ \mathrm{H} & 3.355755 & -0.006637 & -0.585899 \\ \mathrm{H} & 1.182250 & -0.000939 & -1.795297 \\ & -0.946894 & 0.001075 & -0.532177\end{array}$




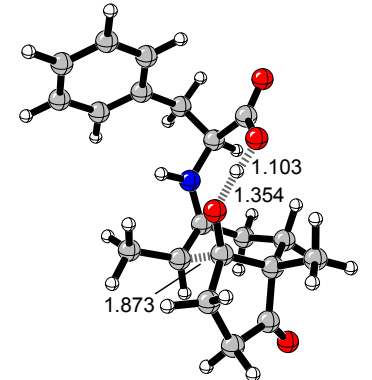

\begin{tabular}{|c|c|c|c|}
\hline \multicolumn{2}{|c|}{$\begin{array}{c}\text { anti-(S,S,R)-12 } \\
\mathrm{E}_{\mathrm{rel}}=0.0 \mathrm{kcal} / \mathrm{mol} \\
\left(\mathrm{E}_{\mathrm{act}}=10.5 \mathrm{kcal} / \mathrm{mol}\right)\end{array}$} & \multicolumn{2}{|c|}{ (Ph gauche-in) } \\
\hline \multicolumn{4}{|c|}{50} \\
\hline $\mathrm{SCF}$ & $=-1132.812518$ & $/ / \mathrm{ZPE}=$ & 19719 \\
\hline $\mathrm{C}$ & 1.887723 & -0.687398 & 0.745901 \\
\hline $\mathrm{C}$ & 1.282670 & -1.370029 & -0.889916 \\
\hline $\mathrm{C}$ & 0.682594 & -0.148092 & -1.322546 \\
\hline $\mathrm{C}$ & 1.605020 & 0.910722 & -1.839939 \\
\hline $\mathrm{C}$ & 2.349126 & 1.530644 & -0.623991 \\
\hline $\mathrm{C}$ & 2.871518 & 0.479973 & 0.364013 \\
\hline 0 & 0.831951 & -0.438385 & 1.468158 \\
\hline $\mathrm{H}$ & 0.161671 & 0.727656 & 1.303742 \\
\hline 0 & -0.357086 & 1.698412 & 1.377883 \\
\hline $\mathrm{C}$ & -1.196262 & 2.122976 & 0.474426 \\
\hline 0 & -1.906726 & 3.106344 & 0.601579 \\
\hline $\mathrm{C}$ & -1.268150 & 1.395783 & -0.915349 \\
\hline $\mathrm{N}$ & -0.560843 & 0.118117 & -0.952636 \\
\hline $\mathrm{H}$ & 1.087458 & 1.690836 & -2.404419 \\
\hline $\mathrm{H}$ & 2.332676 & 0.444121 & -2.509995 \\
\hline $\mathrm{H}$ & 1.675794 & 2.211619 & -0.096162 \\
\hline $\mathrm{H}$ & 3.195974 & 2.115310 & -0.998766 \\
\hline $\mathrm{C}$ & 4.090396 & -0.278975 & -0.166591 \\
\hline 0 & 4.880961 & 0.143194 & -0.985756 \\
\hline C & 4.141035 & -1.673158 & 0.472507 \\
\hline $\mathrm{C}$ & 2.824228 & -1.819836 & 1.253920 \\
\hline $\mathrm{C}$ & 3.300367 & 1.204927 & 1.668847 \\
\hline $\mathrm{H}$ & 5.032767 & -1.753063 & 1.105008 \\
\hline $\mathrm{H}$ & 4.266719 & -2.414688 & -0.325157 \\
\hline $\mathrm{H}$ & 2.368115 & -2.807745 & 1.167392 \\
\hline $\mathrm{H}$ & 2.969896 & -1.637758 & 2.324195 \\
\hline $\mathrm{H}$ & 3.793788 & 0.531458 & 2.376737 \\
\hline $\mathrm{H}$ & 2.414763 & 1.618970 & 2.159045 \\
\hline $\mathrm{H}$ & 3.996529 & 2.017531 & 1.435643 \\
\hline $\mathrm{H}$ & 2.167812 & -1.621815 & -1.473529 \\
\hline C & 0.391440 & -2.561202 & -0.572421 \\
\hline $\mathrm{H}$ & 0.983877 & -3.475522 & -0.483329 \\
\hline $\mathrm{H}$ & -0.352879 & -2.736250 & -1.360038 \\
\hline $\mathrm{H}$ & -0.131103 & -2.408318 & 0.379272 \\
\hline $\mathrm{H}$ & -1.064740 & -0.640685 & -0.500509 \\
\hline $\mathrm{H}$ & -0.778812 & 2.077289 & -1.616107 \\
\hline $\mathrm{C}$ & -2.734292 & 1.212542 & -1.375855 \\
\hline $\mathrm{H}$ & -2.730135 & 0.955027 & -2.441160 \\
\hline $\mathrm{H}$ & -3.205767 & 2.192360 & -1.266772 \\
\hline $\mathrm{C}$ & -3.500059 & 0.159386 & -0.598388 \\
\hline $\mathrm{C}$ & -4.035930 & 0.443587 & 0.668299 \\
\hline $\mathrm{C}$ & -4.719572 & -0.538471 & 1.384765 \\
\hline C & -4.880912 & -1.819483 & 0.851205 \\
\hline C & -4.356363 & -2.113595 & -0.407319 \\
\hline C & -3.671745 & -1.129717 & -1.124801 \\
\hline $\mathrm{H}$ & -3.913082 & 1.438316 & 1.086732 \\
\hline $\mathrm{H}$ & -5.129816 & -0.300826 & 2.362485 \\
\hline $\mathrm{H}$ & -5.416401 & -2.581197 & 1.411361 \\
\hline $\mathrm{H}$ & -4.484819 & -3.104135 & -0.835868 \\
\hline $\mathrm{H}$ & -3.281556 & -1.358851 & -2.114843 \\
\hline
\end{tabular}

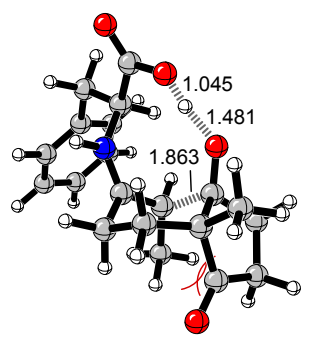

syn-(R,R,R)-12 (Ph anti) $E_{\text {rel }}=7.9 \mathrm{kcal} / \mathrm{mol}$ 50 $\mathrm{SCF}=-1132.801347 / / \mathrm{ZPE}=0.421226$ 1.869615 0.015854 $-0.862892$ 0.399270 0.108096 1. 010940 2. 302904 2.882080 1.397136 0.819351 0.337757 $-0.950650$ $-1.735454$ $-1.417550$ $-0.669335$ 0.561157 1. 251230 2.075792 3.058398 3.544686 3. 974967 3.546680 2.562596 3.969356 4.568487 3. 284538 3.080948 1. 833851 4. 505576 4. 702328 3.491487 $-0.272278$ 0.569538 1. 321602 0.854050 $-0.383353$ $-0.679776$ $-1.097997$ $-2.936656$ $-3.209299$ $-3.411382$ $-3.420190$ $-3.625488$ $-4.036968$ $-4.253388$ $-4.058988$ $-3.645718$ $-3.469302$ $-4.195926$ $-4.579135$ $-4.235438$ $-3.507835$
$-0.935294$ $-0.118773$

$-0.219056$ 0.553608 0.139509 1. 093274 2. 323174 3. 210368 3. 045501 3. 953250 1. 559246 0.956865 0.232632 $-1.256159$ 1. 626162 0.384378 $-1.236164$ $-1.786235$ $-1.835074$ $-0.981038$ 1.168427 $-1.795691$ $-2.895239$ $-0.354673$ $-1.574178$ 0.865123 1.290827 2. 129990 $-0.770621$ $-2.437899$ $-2.686318$ $-2.937396$ $-2.878317$ 1. 521387 1.061505 1. 435095 1. 912425 2.034440 0.001620 $-0.641477$ $-1.973175$ $-2.687796$ $-2.058960$ $-0.725811$ $-0.089141$ $-2.450683$ $-3.723663$ $-2.603068$ $-0.240139$
$-0.227212$

0.908287

2.100600

1. 705980

0.347255

$-1.408585$

$-0.818683$

$-0.550245$

$-0.327072$

$-0.140327$

$-0.245215$

2. 992638

2. 337563

1. 683869

2. 480828

0.383944

$-1.025943$

$-1.847424$

$-0.060704$

$-1.423655$

$-0.958664$

$-2.579938$

$-2.407040$

$-0.965563$

0.744149

$-0.268460$

$-1.067796$

0.017024

0.769169

$-0.912862$

0.335460

1. 716354

$-1.161442$

$-0.042900$

0.904795

$-0.089159$

$-1.318911$

$-0.191838$

1. 038722

1.086645

$-2.243538$

$-2.335721$

$-0.231434$

1. 962997

2. 050306
0.871331

1. 378983

$-0.826402$

$-1.372452$ 


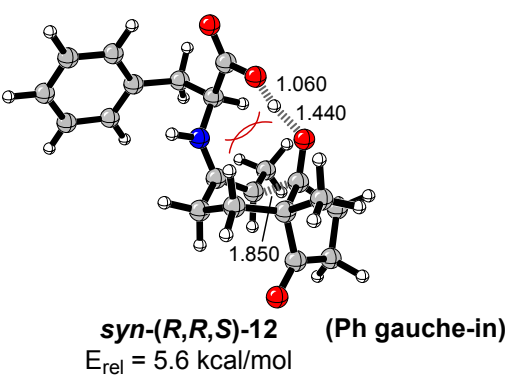
50

\begin{tabular}{|c|c|c|c|}
\hline & -1132.804978 & $/ / \mathrm{ZPE}=0$ & $1 \perp 49$ \\
\hline C & -2.478807 & 0.624841 & 0.308825 \\
\hline C & -1.400907 & -0.427867 & 1.381858 \\
\hline $\mathrm{C}$ & -0.385593 & -0.772843 & 0.434378 \\
\hline C & -0.776254 & -1.788822 & -0.600382 \\
\hline C & -1.690388 & -1.034150 & -1.605590 \\
\hline $\mathrm{C}$ & -2.843831 & -0.291431 & -0.925243 \\
\hline O & -1.869019 & 1.755420 & 0.123545 \\
\hline $\mathrm{H}$ & -0.693246 & 1.970242 & -0.678957 \\
\hline O & 0.163057 & 2.300042 & -1.209050 \\
\hline $\mathrm{C}$ & 1.293930 & 2.176138 & -0.552847 \\
\hline O & 2.314855 & 2.772041 & -0.839907 \\
\hline $\mathrm{C}$ & 1.268729 & 1.175646 & 0.653365 \\
\hline $\mathrm{N}$ & 0.729089 & -0.104779 & 0.170756 \\
\hline $\mathrm{H}$ & 0.095926 & -2.200890 & -1.120980 \\
\hline $\mathrm{H}$ & -1.324076 & -2.619243 & -0.148372 \\
\hline $\mathrm{H}$ & -1.082533 & -0.318874 & -2.172436 \\
\hline $\mathrm{H}$ & -2.103031 & -1.762569 & -2.311874 \\
\hline $\mathrm{C}$ & -3.881493 & -1.254704 & -0.343093 \\
\hline O & -4.107640 & -2.375067 & -0.754783 \\
\hline $\mathrm{C}$ & -4.567285 & -0.607073 & 0.865607 \\
\hline $\mathrm{C}$ & -3.808500 & 0.703779 & 1.121357 \\
\hline $\mathrm{C}$ & -3.560039 & 0.594999 & -1.977464 \\
\hline $\mathrm{H}$ & -5.630973 & -0.458717 & 0.645287 \\
\hline $\mathrm{H}$ & -4.527788 & -1.314306 & 1.702863 \\
\hline $\mathrm{H}$ & -3.634001 & 0.912096 & 2.179029 \\
\hline $\mathrm{H}$ & -4.350271 & 1.567006 & 0.720582 \\
\hline $\mathrm{H}$ & -4.476442 & 1.046286 & -1.583671 \\
\hline $\mathrm{H}$ & -3.827646 & 0.000764 & -2.857575 \\
\hline $\mathrm{H}$ & -2.892479 & 1.408774 & -2.275207 \\
\hline $\mathrm{H}$ & -2.005132 & -1.314141 & 1.579631 \\
\hline C & -1.097528 & 0.350591 & 2.657831 \\
\hline $\mathrm{H}$ & -1.825223 & 0.092042 & 3.432586 \\
\hline $\mathrm{H}$ & -1.166012 & 1.429126 & 2.482209 \\
\hline $\mathrm{H}$ & -0.106121 & 0.118144 & 3.063687 \\
\hline $\mathrm{H}$ & 1.295167 & -0.494898 & -0.577772 \\
\hline $\mathrm{H}$ & 0.565799 & 1.585805 & 1.376129 \\
\hline C & 2.655851 & 0.981993 & 1.309251 \\
\hline $\mathrm{H}$ & 3.119133 & 1.970632 & 1.365000 \\
\hline $\mathrm{H}$ & 2.492629 & 0.631053 & 2.334997 \\
\hline $\mathrm{C}$ & 3.575809 & 0.002944 & 0.598573 \\
\hline $\mathrm{C}$ & 4.313606 & 0.375192 & -0.536784 \\
\hline C & 5.143932 & -0.547072 & -1.176287 \\
\hline $\mathrm{C}$ & 5.252198 & -1.854383 & -0.697703 \\
\hline $\mathrm{C}$ & 4.524362 & -2.236307 & 0.430076 \\
\hline $\mathrm{C}$ & 3.694271 & -1.313719 & 1.069394 \\
\hline $\mathrm{H}$ & 4.225587 & 1.389020 & -0.912555 \\
\hline $\mathrm{H}$ & 5.711534 & -0.239254 & -2.050593 \\
\hline $\mathrm{H}$ & 5.902363 & -2.568195 & -1.196535 \\
\hline $\mathrm{H}$ & 4.605826 & -3.248860 & 0.816912 \\
\hline $\mathrm{H}$ & 3.138719 & -1.615987 & 1.955020 \\
\hline
\end{tabular}

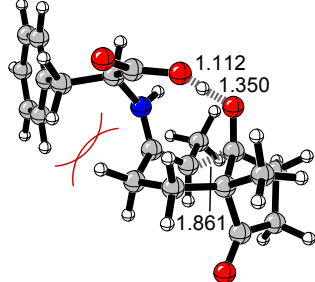

$\quad \begin{aligned} & \text { anti-( } \boldsymbol{R}, \boldsymbol{R}, \boldsymbol{S})-\mathbf{1 2} \\ & \mathrm{E}_{\mathrm{rel}}=5.4 \mathrm{kcal} / \mathrm{mol}\end{aligned}$
50
$\mathrm{SCE}=-1132.803593 / / \mathrm{ZPE}=0.419470$

$\mathrm{SCF}=-\begin{array}{cc}-1132.803593 \\ 0.283152 & -0.446961\end{array}$

\begin{tabular}{|c|c|c|c|}
\hline $\mathrm{C}$ & 0.283152 & -0.446961 & -1.814062 \\
\hline $\mathrm{C}$ & 0.300813 & -0.441525 & -0.383602 \\
\hline $\mathrm{C}$ & 1.647279 & -0.477850 & 0.272426 \\
\hline $\mathrm{C}$ & 2.435222 & 0.835472 & -0.031737 \\
\hline $\mathrm{C}$ & 2.178155 & 1.441115 & -1.417859 \\
\hline $\mathrm{C}$ & 0.713358 & 1.355383 & -1.983322 \\
\hline 0 & -0.250222 & 2.049872 & -1.435018 \\
\hline $\mathrm{H}$ & -0.186935 & 2.230148 & -0.098520 \\
\hline 0 & -0.022785 & 2.506260 & 0.965765 \\
\hline $\mathrm{C}$ & -0.232395 & 1.666009 & 1.946479 \\
\hline 0 & 0.099183 & 1.859620 & 3.102623 \\
\hline C & -1.045307 & 0.373043 & 1.615039 \\
\hline $\mathrm{N}$ & -0.817893 & -0.112458 & 0.242713 \\
\hline $\mathrm{H}$ & -1.622765 & -0.026645 & -0.365681 \\
\hline $\mathrm{H}$ & -2.092620 & 0.697783 & 1.605880 \\
\hline $\mathrm{C}$ & -0.921533 & -0.721235 & 2.695019 \\
\hline $\mathrm{H}$ & -1.064034 & -0.209796 & 3.650638 \\
\hline $\mathrm{H}$ & 0.097598 & -1.119238 & 2.712971 \\
\hline $\mathrm{C}$ & -1.921954 & -1.843175 & 2.517562 \\
\hline $\mathrm{C}$ & -3.250174 & -1.684397 & 2.939665 \\
\hline $\mathrm{C}$ & -4.187210 & -2.701285 & 2.756736 \\
\hline C & -3.810100 & -3.900767 & 2.148719 \\
\hline $\mathrm{C}$ & -2.490738 & -4.074821 & 1.729256 \\
\hline C & -1.557238 & -3.052740 & 1.912339 \\
\hline $\mathrm{H}$ & -0.528326 & -3.201806 & 1.591761 \\
\hline $\mathrm{H}$ & -2.184921 & -5.008196 & 1.263765 \\
\hline $\mathrm{H}$ & -4.537657 & -4.695892 & 2.010399 \\
\hline $\mathrm{H}$ & -5.209798 & -2.560493 & 3.096879 \\
\hline $\mathrm{H}$ & -3.549877 & -0.758393 & 3.426870 \\
\hline $\mathrm{H}$ & 1.587413 & -0.619550 & 1.352613 \\
\hline $\mathrm{H}$ & 2.193564 & -1.329987 & -0.145371 \\
\hline $\mathrm{H}$ & 2.187094 & 1.575357 & 0.729892 \\
\hline $\mathrm{H}$ & 3.504054 & 0.612329 & 0.052439 \\
\hline $\mathrm{C}$ & 2.990593 & 0.760714 & -2.525268 \\
\hline $\mathrm{C}$ & 2.256254 & 0.912504 & -3.864465 \\
\hline $\mathrm{C}$ & 0.889897 & 1.526213 & -3.517570 \\
\hline 0 & 4.040758 & 0.172600 & -2.368991 \\
\hline $\mathrm{H}$ & 2.853634 & 1.534958 & -4.540601 \\
\hline $\mathrm{H}$ & 2.190113 & -0.074210 & -4.337614 \\
\hline $\mathrm{H}$ & 0.058206 & 1.102404 & -4.083182 \\
\hline $\mathrm{H}$ & 0.874612 & 2.605016 & -3.705904 \\
\hline $\mathrm{C}$ & 2.610878 & 2.933297 & -1.380756 \\
\hline $\mathrm{H}$ & 2.575942 & 3.402024 & -2.369228 \\
\hline $\mathrm{H}$ & 3.635927 & 3.016296 & -1.004289 \\
\hline $\mathrm{H}$ & 1.939916 & 3.484764 & -0.716778 \\
\hline $\mathrm{H}$ & 1.116075 & -1.012408 & -2.231346 \\
\hline $\mathrm{C}$ & -1.036863 & -0.681677 & -2.534933 \\
\hline $\mathrm{H}$ & -0.866134 & -0.898278 & -3.592382 \\
\hline $\mathrm{H}$ & -1.674843 & 0.209272 & -2.479943 \\
\hline $\mathrm{H}$ & -1.584278 & -1.537142 & -2.117682 \\
\hline
\end{tabular}




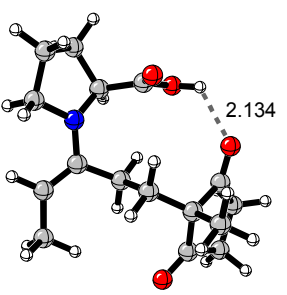

E-anti-a (O-H-O) 0.0

44

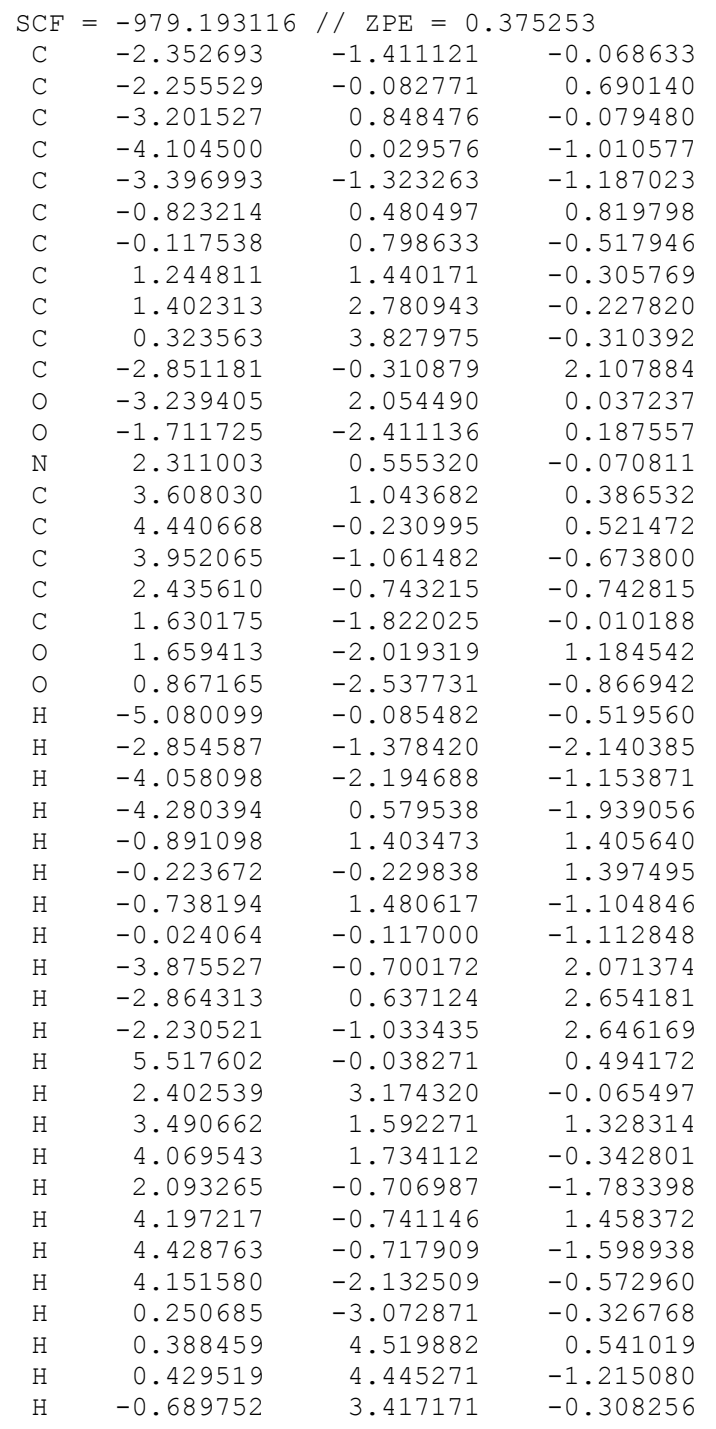

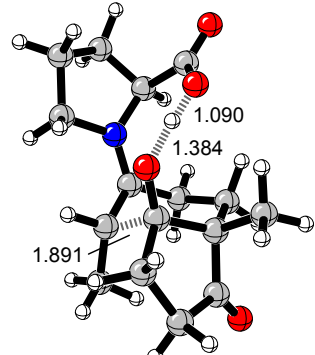

$(S, S, S)-13$

$E_{\text {rel }}=0.0 \mathrm{kcal} / \mathrm{mol}$ $\left(\mathrm{E}_{\mathrm{act}}=15.7 \mathrm{kcal} / \mathrm{mol}\right)$ 44

$\mathrm{SCF}=-979.168111 / / \mathrm{ZPE}=0.375231$

$\mathrm{C}$

$-1.184837$

$-0.683459$

0.322772

$-0.169763$

$-0.839033$

$-1.713008$

$-0.155939$

0.845756

1. 581571

2. 502953

3. 345482

2.568805

1.549361

0.624441

$-0.906256$

$-1.460572$

$-0.059131$

$-3.112807$

$-3.697418$

$-3.663627$

$-2.452903$

$-1.913328$

$-4.425179$

$-4.179950$

$-2.367242$

$-2.500019$

$-2.603146$

$-0.951910$

$-2.317521$

2. 104511

3.568906

3.888670

1. 529508

2. 020187

4. 228142

3. 660930

4.116688

4.710442

2. 473546

$-0.291398$

$-1.782434$

$-2.498289$

$-1.358207$

$-2.345425$

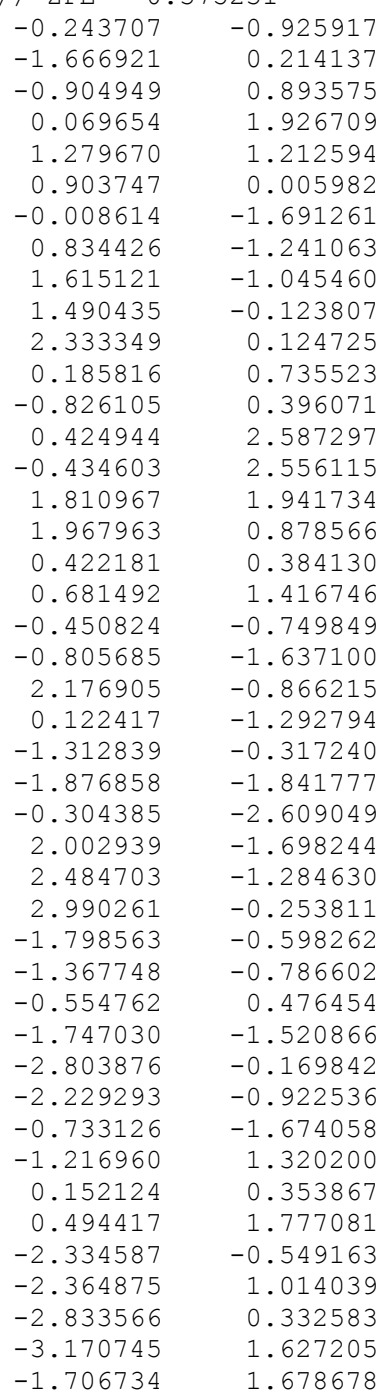




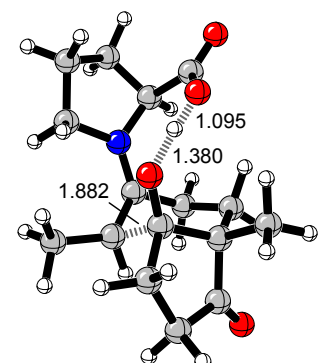

$(S, S, R)-13$

$E_{\text {rel }}=1.3 \mathrm{kcal} / \mathrm{mol}$ 44

$\mathrm{SCF}=-979.166169 / / \mathrm{ZPE}=0.375444$

$\begin{array}{rrrr}\mathrm{C} & 1.271208 & 0.401696 & -0.790859 \\ \mathrm{C} & 0.774979 & 1.522465 & 0.636667 \\ \mathrm{C} & -0.287949 & 0.665262 & 1.086005 \\ \mathrm{C} & 0.210043 & -0.503260 & 1.902722 \\ \mathrm{C} & 0.893512 & -1.537624 & 0.968598 \\ \mathrm{C} & 1.806670 & -0.898764 & -0.084784 \\ \mathrm{O} & 0.262066 & 0.325788 & -1.612971 \\ \mathrm{H} & -0.732987 & -0.594853 & -1.355918 \\ \mathrm{O} & -1.462289 & -1.410909 & -1.335386 \\ \mathrm{C} & -2.371991 & -1.531917 & -0.402461 \\ \mathrm{O} & -3.174667 & -2.445425 & -0.339139 \\ \mathrm{C} & -2.486969 & -0.449261 & 0.722292 \\ \mathrm{~N} & -1.520343 & 0.664366 & 0.596050 \\ \mathrm{H} & -0.578557 & -0.988654 & 2.481293 \\ \mathrm{H} & 0.946511 & -0.125978 & 2.617369 \\ \mathrm{H} & 1.491838 & -2.216387 & 1.586208 \\ \mathrm{H} & 0.130474 & -2.134044 & 0.463614 \\ \mathrm{C} & 3.144686 & -0.431313 & 0.493913 \\ \mathrm{O} & 3.689512 & -0.895037 & 1.474873 \\ \mathrm{C} & 3.679900 & 0.739933 & -0.341319 \\ \mathrm{C} & 2.556666 & 1.087094 & -1.331655 \\ \mathrm{C} & 2.116210 & -1.962722 & -1.173759 \\ \mathrm{H} & 4.616116 & 0.445364 & -0.829371 \\ \mathrm{H} & 3.936188 & 1.560647 & 0.339327 \\ \mathrm{H} & 2.739940 & 0.642485 & -2.315911 \\ \mathrm{H} & 2.426642 & 2.157442 & -1.498186 \\ \mathrm{H} & 2.866056 & -1.618163 & -1.893015 \\ \mathrm{H} & 2.494118 & -2.877921 & -0.705817 \\ \mathrm{H} & 1.199525 & -2.192714 & -1.723663 \\ \mathrm{C} & -3.846327 & 0.254808 & 0.632003 \\ \mathrm{C} & -3.591126 & 1.307739 & -0.453816 \\ \mathrm{C} & -2.150460 & 1.776356 & -0.190050 \\ \mathrm{H} & -4.633794 & -0.457811 & 0.383505 \\ \mathrm{H} & -4.087743 & 0.728522 & 1.591145 \\ \mathrm{H} & -3.661498 & 0.847261 & -1.444608 \\ \mathrm{H} & -4.296342 & 2.142710 & -0.422004 \\ \mathrm{H} & -1.588779 & 1.934105 & -1.106463 \\ \mathrm{H} & -2.129192 & 2.681794 & 0.420555 \\ \mathrm{H} & -2.360557 & -0.968559 & 1.671628 \\ \mathrm{C} & 0.610941 & 2.948588 & 0.118326 \\ \mathrm{H} & 1.597553 & 1.475075 & 1.350459 \\ \mathrm{H} & 1.587142 & 3.442356 & 0.120908 \\ \mathrm{H} & 0.228967 & 2.981439 & -0.904242 \\ \mathrm{H} & -0.041937 & 3.551710 & 0.757985\end{array}$

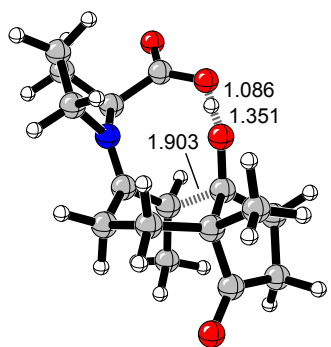

$(R, R, R)-13$ $\mathrm{E}_{\mathrm{rel}}=3.7 \mathrm{kcal} / \mathrm{mol}$ 44

$\mathrm{SCF}=-979.161849 / / \mathrm{ZPE}=0.374857$

$-1$

$-0.303752$

0.312715

$-0.589038$

$-1.517756$

$-2.126117$

$-0.183710$

0.938829

1.836649

2.815446

3.907383

2. 619006

1.528817

$-0.030141$

$-1.203230$

$-0.944952$

$-2.322849$

$-3.294385$

$-4.023888$

$-3.391060$

$-2.049817$

$-2.661363$

$-4.246893$

$-3.615500$

$-2.173029$

$-1.582438$

$-3.213070$

$-3.332403$

$-1.810804$

2.065722

3.547428

3.868734

1.952870

1.501859

3.684996

4.165637

4.759387

3.973941

2. 451367

$-1.179154$

0.371936

$-1.981993$

$-1.636500$

$-0.566583$ 


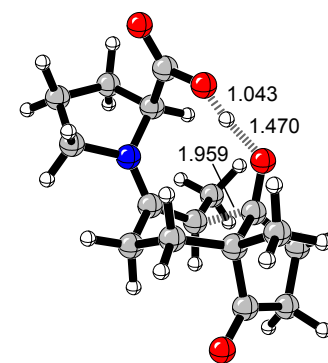

$(R, R, S)-13$

$\mathrm{E}_{\mathrm{rel}}=2.9 \mathrm{kcal} / \mathrm{mol}$

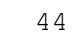

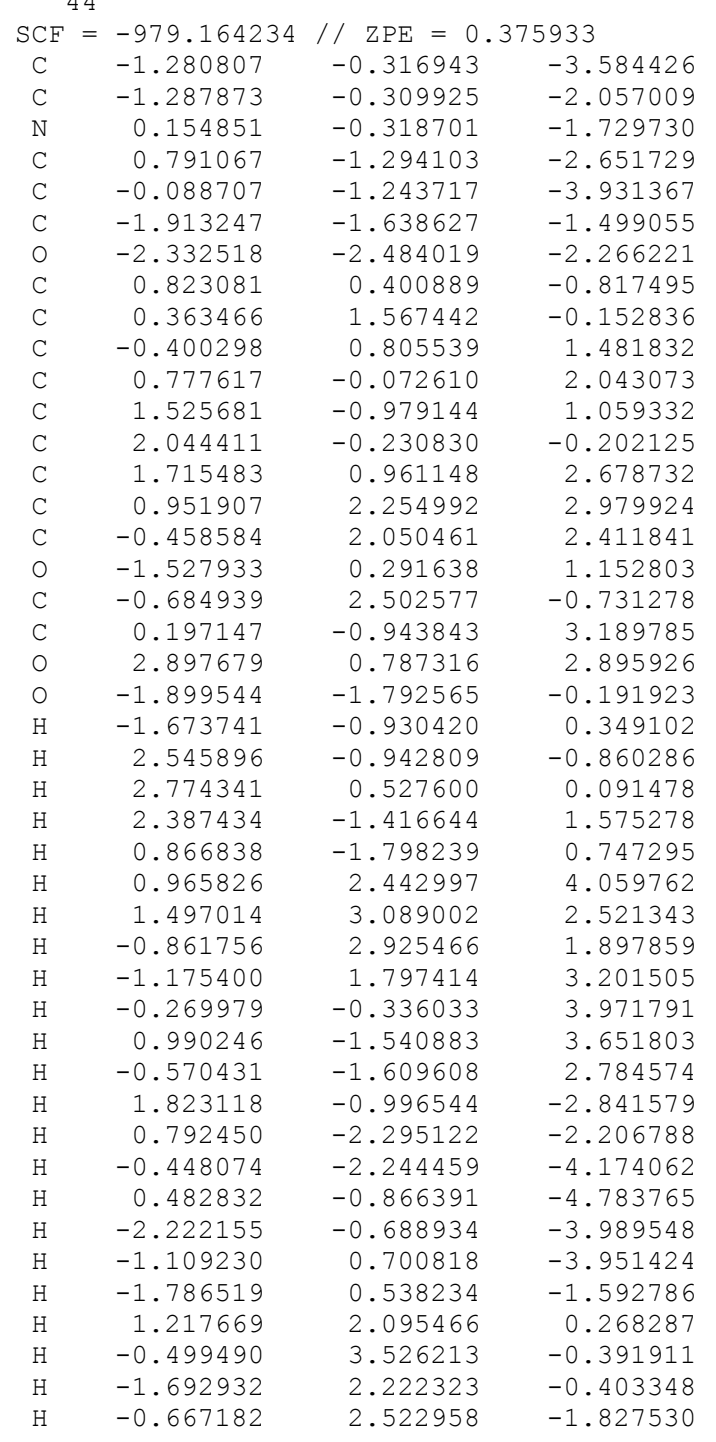

